\title{
SOME MODEL THEORY OF MODULES OVER BÉZOUT DOMAINS. THE WIDTH.
}

\author{
GENA PUNINSKI AND CARLO TOFFALORI
}

\begin{abstract}
We will develop the model theory of modules over commutative Bézout domains. In particular we characterize commutative Bézout domains $B$ whose lattice of pp-formulae has no width and give some applications to the existence of superdecomposable pure injective $B$-modules.
\end{abstract}

\section{INTRODUCTION}

An idealistic approach to model theory of modules over a ring $R$ would be through a complete description of the lattice $L(R)$ of pp-formulae in one variable over $R$. Having achieved this goal, the description of the Ziegler spectrum $\mathrm{Zg}_{R}$ over $R$ is usually not difficult, and various model theoretic dimensions (like $m$-dimension, breadth and width) would admit straightforward calculations.

Unfortunately, a complete description of $L(R)$ is very rarely available: even over rings of integers it causes some difficulties, but over many rings (like string algebras — see [10]) is hardly possible. Thus various indirect methods were developed to calculate the above mentioned model theoretic invariants.

However there is one class of rings, where this program has been successfully carried through - commutative valuation domains $V$. In this case (see [8]) the whole lattice $L(V)$ is a free product of two copies of the positive cone $\Gamma^{+}(V)$ of the value group of $V$, and after that all these dimensions, and the Ziegler spectrum of $B$ are calculated without much effort. The advantage of this approach is that many natural questions on $\mathrm{Zg}_{V}$ and on the theory $T(V)$ of $V$-modules admit a natural interpretation and can be tackled very effectively. For instance, the question on decidability of $T(V)$ was answered almost completely (see [12], [3], [5]) and the sobriety of $\mathrm{Zg}_{V}$ (see [4]) was

2000 Mathematics Subject Classification. 03C60.

Key words and phrases. Bézout domain, commutative valuation domain, width of the lattice of pp-formulas, superdecomposable pure injective module.

The second author is supported by Italian PRIN. 
established. Furthermore (see [9, Ch. 12]) a superdecomposable pure injective $V$-module exists if and only if $\Gamma^{+}(V)$ contains a dense subchain if and only if the width of $L(V)$ is undefined.

In this paper we will extend this natural approach to commutative Bézout domains $B$. This class of rings is undoubtedly of great importance in algebra, including among others the ring of algebraic integers and the ring of entire (complex or real) functions in one variable. We will describe, in terms of the positive cone $\Gamma^{+}(B)$ of the value group $\Gamma(B)$ of $B$, the whole lattice $L(B)$. Despite being quite technical and lengthy, this result is expected to be very useful for future applications. Due to a sheer volume of the paper we will restrict ourselves with just a few. For instance, we will give a syntactical description, in a spirit of original Ziegler [14], of the Ziegler spectrum of $B$. A basis for topology is very similar to what is known for commutative valuation domains and given by a combination of simple divisibility and annihilator conditions. The points of $\mathrm{Zg}_{B}$ (that is, indecomposable pure injective $B$-modules) are described by pairs of filter-cofilter partitions on $\Gamma^{+}(B)$, with a precise criterion when two pairs produce the same point.

We will also approach an old and unresolved question on the equivalence of the existence of a superdecomposable pure injective $B$-module and the non-existence of the width of $L(B)$. Unable to give a complete answer we will prove that the width of this lattice is undefined if and only if the lattice ordered group $\Gamma(B)$ contains a dense subchain. Surprisingly we will see that the unresolved implication (no width implies a superdecomposable pure injective) is quite difficult even in the torsion free (or divisible) case.

As we will show in this paper, almost all problems in model theory of modules over commutative Bézout domains are in fact questions about lattice ordered abelian groups. Therefore these model theoretic problems appear to be a good test for applying well developed methods of this classical part of algebra.

We hope that this paper will be just a first step in a program of investigating model theoretic properties of modules over very interesting examples of rings originated in algebra and analysis.

\section{Preliminaries}

All rings $R$ in this paper will be commutative rings with unity, and all modules $M$ are unitary (and mostly right) $R$-modules. 
Recall that a ring $R$ is said to be Prüfer, if the lattice of ideals of $R$ is distributive. For instance, $R$ is Prüfer if and only if for every prime (or maximal) ideal $P$, the localization $R_{P}$ is a (commutative) valuation ring, that is all ideals of $R_{P}$ are linearly ordered by inclusion. For various characterizations of Prüfer domains (for instance, this property is equivalent to semiheredity) see [1, Ch. 3]. But the following Warfield's result holds even for rings with zero divisors.

Fact 2.1. [13, Thm. 1] Every finitely presented module over a Prüfer ring $R$ is a direct summand of a (finite) direct sum of modules $R / r R, r \in R$.

One consequence of this result for model theory of modules is straightforward.

Recall that a positive primitive formula (pp-formula) $\varphi(x)$ in one variable $x$ is an existentially quantified formula $\exists \bar{y}=\left(y_{1}, \ldots, y_{n}\right) \bar{y} A=x \bar{b}$, where $\bar{b}=\left(b_{1}, \ldots, b_{k}\right)$ is a row of elements of $R$ and $A$ is an $n \times k$ matrix over $R$. If $M$ is an $R$-module and $m \in M$, then we say that $m$ satisfies $\varphi$ in $M$, written $M \models \varphi(m)$, if there is a tuple $\bar{m}=\left(m_{1}, \ldots, m_{n}\right) \in M$ such that $\bar{m} A=m \bar{b}$ (that is, if the corresponding system of linear equations is soluble). By $\varphi(M)$ we will denote the submodule $\{m \in M \mid M \models \varphi(m)\}$ defined by $\varphi$ in $M$.

We say that pp-formulas $\varphi(x)$ and $\psi(x)$ are equivalent if $\varphi(M)=\psi(M)$ in any module $M$. For an algebraic description of this equivalence relation, in terms of matrix equations, see [7, L. 1.1.13]. Actually this equivalence relation applies to pairs of arbitrary formulas. It turns out that, up to this relation, if $\varphi(x), \psi(x)$ are pp-formulas, then also their conjunction $\varphi(x) \wedge$ $\psi(x)$ and their sum $\varphi(x)+\psi(x) \doteq \exists u \exists v(\varphi(u) \wedge \psi(v) \wedge x=u+v)$ are ppformulas. The following property, coined as RD-property in [11], is a direct consequence of Fact 2.1.

Fact 2.2. Every pp-formula $\varphi(x)$ over a Prüfer ring $R$ is equivalent to a finite sum of formulas $\exists y(y a=x \wedge y b=0)$, and also to a finite conjunction of formulae $c \mid x d$, that is $\exists y(y c=x d)$.

We say that a ring $R$ is Bézout, if every 2-generated ideal of $R$ is principal (and therefore every finitely generated ideal of $R$ is principal). For instance, every Bézout ring is Prüfer. In this paper we will deal mostly with Bézout domains, and we will use the letter $B$ to denote such rings. Thus a domain $B$ is Bézout if and only if for any $a, b \in B$ there exist $u, v, s, t \in B$ such that $(a u+b v) s=a$ and $(a u+b v) t=b$, that is $c=a u+b v$ is a single generator 
for the ideal $a B+b B$. These identities are often called Bézout identities. Note that the element $c$ as above is defined up to multiplication by a unit, and called the greatest common divisor of $a$ and $b, \operatorname{gcd}(a, b)$.

Localizing, it is not difficult to check that in a Bézout domain $B$ the intersection $a B \cap b B$ of principal ideals is also a principal ideal $d R$ (that is, every Bézout domain is coherent), and we call this $d$ the least common multiple of $a$ and $b, \operatorname{lcm}(a, b)$ (again, $d$ is defined up to a multiplicative unit). For instance, it is easily shown that $a b=\operatorname{gcd}(a, b) \cdot \operatorname{lcm}(a, b)$.

For more properties of Bézout domains the reader is referred to [1, Sec. 3.5]. For instance, every principal ideal domain is Bézout. Furthermore (see [1, Exam. 3.1.3]) the ring of algebraic integers is Bézout, so as (see [6]) the ring of entire complex or real functions in one variable.

Recall that a divisibility formula is a pp-formula of the form $a \mid x \doteq$ $\exists y(y a=x), a \in B$, therefore $(a \mid x)(M)=M a$ for any module $M$. Similarly, the annihilator formula $x b=0$ defines in any module $M$ the annihilator of $b: \operatorname{ann}_{M}(b) \doteq\{m \in M \mid m b=0\}$.

Over Bézout domains every pp-formula is equivalent to a Boolean combination of divisibility and annihilator conditions.

Lemma 2.3. Let $B$ be a Bézout domain. Then every pp-formula over $B$ is equivalent to

1) a finite conjunction of formulas $\varphi_{a, b} \doteq a \mid x+x b=0$, and also to

2) a finite sum of formulas $\psi_{c, d} \doteq c \mid x \wedge x d=0$.

Proof. We will prove just 1), and then 2) will follow by duality (see an explanation at the end of this section).

By Fact 2.2 it suffices to consider the formula $\chi \doteq a \mid x b \doteq \exists y(y a=x b)$. Let $c=\operatorname{gcd}(a, b)$, therefore $a=c a^{\prime}$ and $b=c b^{\prime}$, where $a^{\prime}$ and $b^{\prime}$ are coprime, that is $a^{\prime} u+b^{\prime} v=1$ for some $u, v \in B$. We will show that $\chi$ is equivalent to $\varphi_{a^{\prime}, c} \doteq a^{\prime} \mid x+x c=0$.

Indeed, suppose first that $m \in \varphi_{a^{\prime}, c}(M)$, therefore $m=n a^{\prime}+k$, where $k c=0$. Multiplying by $c$ we obtain $m c=n a^{\prime} c=n a$, therefore $m b=m c b^{\prime}=$ $n a b^{\prime} \in M a$. It follows that $\varphi_{a^{\prime}, c}$ implies $\chi$.

Now assume that $M=\chi(m)$, that is $m b=n a$ for some $n \in M$. Then $m=m a^{\prime} u+m b^{\prime} v=\left(m a^{\prime} u+n a^{\prime} v\right)+\left(m b^{\prime} v-n a^{\prime} v\right)$, where $m a^{\prime} u+n a^{\prime} v \in M a^{\prime}$ and $\left(m b^{\prime} v-n a^{\prime} v\right) c=(m b-n a) v=0$. It follows that $\chi$ implies $\varphi_{a^{\prime}, c}$. 
Note that the formula $\varphi_{a, b}$ is equivalent to the formula $a b \mid x b$, since $(a b \mid x b)(M)=\{m \in M \mid m b \in M a b\}$ clearly equals $M a+\operatorname{ann}_{M}(b)$ in any module $M$.

If $\varphi$ and $\psi$ are pp-formulae, we say that $\varphi$ implies $\psi$, written $\varphi \rightarrow \psi$, if $\varphi(M) \subseteq \psi(M)$ for any module $M$ (actually we already used this notion in the proof of Lemma 2.3). For instance, it is straightforward to check that, over any ring $R$, we have $a\left|x \rightarrow a^{\prime}\right| x$ iff $a \in R a^{\prime}$ and $x b=0 \rightarrow x b^{\prime}=0$ iff $b^{\prime} \in b R$. Furthermore, if $R$ is a domain and $0 \neq a, b \in R$, then $a \mid x \rightarrow x b=0$ is never true; and $x b=0 \rightarrow a \mid x$ holds if and only if $1=b s+t a$ for some $s, t \in R$.

If we factorize the set of all pp-formulae in one variable over a ring $R$ identifying equivalent formulae, we obtain a set $L(R)$. Furthermore the implication defines a partial ordering on $L(R)$ such that $L(R)$ is a lattice: the meet is given by conjunction of formulae, and the join is formed by the sum of formulae.

The following useful remark can also be derived from Fact 2.1.

Fact 2.4. If $R$ is a Prüfer ring, then the lattice $L(R)$ is distributive.

Note that above we defined the right pp-formulae over a ring $R$. A similar definition gives also left pp-formulae over $R$. By [7, Sec. 1.3.1]) there exists a duality, called elementary duality, between lattices of left and right ppformulae, which interchanges divisibility and annihilator conditions.

\section{PP-Formulae}

If $B$ is a Bézout domain, then Lemma 2.3 claims that the lattice $L(B)$ is generated by divisibility and annihilator conditions. The following lemma shows that the result of lattice operations on generators is natural.

Lemma 3.1. Let $B$ be a Bézout domain and $a, b, a^{\prime}, b^{\prime} \in R$. The the following holds:

1) $a\left|x+a^{\prime}\right| x$ is equivalent to $c \mid x$, where $c=\operatorname{gcd}\left(a, a^{\prime}\right)$.

2) $a\left|x \wedge a^{\prime}\right| x$ is equivalent to $l \mid x$, where $l=\operatorname{lcm}\left(a, a^{\prime}\right)$.

3) $x b=0+x b^{\prime}=0$ is equivalent to $x d=0$, where $d=\operatorname{lcm}\left(b, b^{\prime}\right)$.

4) $x b=0 \wedge x b^{\prime}=0$ is equivalent to $x g=0$, where $g=\operatorname{gcd}\left(b, b^{\prime}\right)$.

Proof. By elementary duality it suffices to prove 1) and 3).

1) We have $c=a u+a^{\prime} u^{\prime}$ and also $a=c s, a^{\prime}=c s^{\prime}$ for some $u, u^{\prime}, s, s^{\prime} \in B$. From $a=c s$ it follows $a|x \rightarrow c| x$, and similarly $a^{\prime} \mid x$ implies $c \mid x$; therefore $a\left|x+a^{\prime}\right| x \rightarrow c \mid x$. 
For the converse implication suppose that for some $B$-module $M$ and $m \in M$ we have $M \models(c \mid x)(M)$, that is $m=n c$ for some $n \in M$. Then $m=n c=n\left(a u+a^{\prime} u^{\prime}\right)=n u a+n u^{\prime} a^{\prime} \in M a+M a^{\prime}$, therefore $c \mid x$ implies $a\left|x+a^{\prime}\right| x$.

3) Clearly $d=0$ if and only if $b=0$ or $b^{\prime}=0$, and in this case the conclusion is trivial. Thus we can suppose that $d, b, b^{\prime} \neq 0$. From $d B=$ $b B \cap b^{\prime} B$ it follows that $d=b g=b^{\prime} g^{\prime}$, where $0 \neq g, g^{\prime}$ are coprime (that is, $\left.g B+g^{\prime} B=B\right)$. Thus choose $u, u^{\prime} \in B$ such that $g u+g^{\prime} u^{\prime}=1$.

From $d \in b B, b^{\prime} B$ it follows that $x b=0, x b^{\prime}=0 \rightarrow x d=0$, therefore $x b=0+x b^{\prime}=0$ implies $x d=0$. For the converse we assume that $m d=0$ for some element $m$ of a $B$-module $M$. Then $m=m\left(g u+g^{\prime} u^{\prime}\right)=m g u+m g^{\prime} u^{\prime}$, where $(m g u) b=m d u=0$ and $\left(m g^{\prime} u^{\prime}\right) b^{\prime}=m d u^{\prime}=0$, therefore $x d=0$ implies $x b=0+x b^{\prime}=0$.

If $a, b \in B$, then we define $a \leq b$ if $b \in a B$ (that is $a B \supseteq b B$ ). Intuitively this ordering corresponds to divisibility for integers, say $2 \leq 4$ (but not vice versa), and is opposite to the inclusion relation for principal ideals of $B$. If we set $a \sim b$ for $a \leq b \leq a$, then $a \sim b$ if and only if $a B=b B$. The set of equivalence classes of this relation can be identified with $B$ factorized by units, that is with the set $\Gamma^{+}(B)$ of principal ideals of $B$. Our ordering $\leq$ induces the partial ordering on this set such that $\Gamma^{+}(B)$ is a lattice, with gcd as a meet and $\mathrm{lcm}$ as a join. We illustrate this with the following diagram.

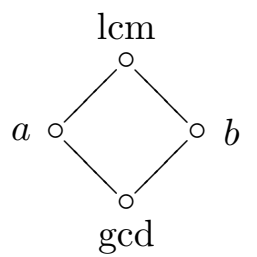

Note that on level of principal ideals we have $a R+b R=\operatorname{gcd}(a, b) R$ and $a R \cap b R=\operatorname{lcm}(a, b) R$, therefore $\Gamma^{+}(B)$ is anti-isomorphic to the lattice of principal ideals. But to avoid reversing the ordering we will prefer to look at this lattice as a set of (equivalence classes of) elements of $B$, rather than principal ideals. In this sense we will sometimes identify the elements of $B$ and their equivalence classes in $\Gamma^{+}(B)$ (or the corresponding principal ideals).

Clearly $\Gamma^{+}(B)$ is a non-negative part of the value group of $B$, which is an abelian lattice ordered group (see [1, Sec. 3.5] for more on that). Furthermore, the famous Kaplansky-Jaffard-Ohm theorem (see [1, Thm. 3.5.3]) says that every lattice ordered abelian groups occurs as a value group of 
some Bézout domain $B$. As we will see, the model theoretical properties of $B$, in particular its lattice $L(B)$, depend only on $\Gamma^{+}(B)$.

From Lemma 3.1 it follows that the lattice $L(B)$ is generated by two copies of $\Gamma^{+}(B)$ - one formed by divisibility formulae, and another by annihilator conditions.

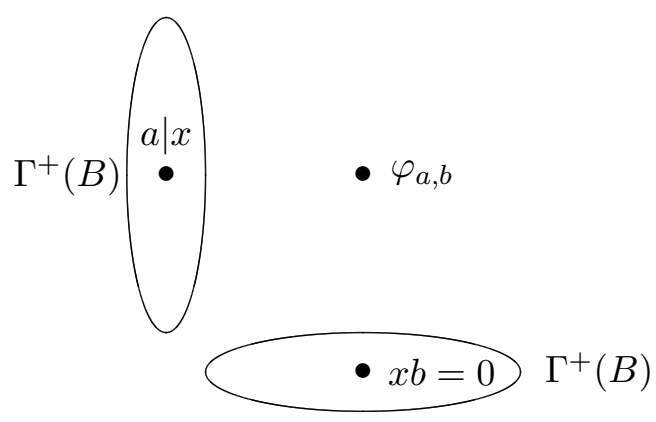

Here we show a formula $\varphi_{a, b} \doteq a \mid x+x b=0$ as a point on the $\Gamma^{+}(B) \times$ $\Gamma^{+}(B)$ plane such that the divisibility formulae correspond to the first (that is, vertical) coordinate and the annihilator conditions to the second (that is, horizontal) coordinate. Note that in the previous diagram both the vertical and the horizontal axis are denoted by encircled regions rather than by lines. This is to underline that $\Gamma^{+}(B)$ is a lattice, but not necessarily a total order. By Lemma 2.3 every pp-formula over a Bézout domain $B$ is equivalent to a finite conjunction of such formulae, therefore it is shown as a finite number of points on this plane (connected by 'invisible' conjunctions).

Our next goal is to investigate implications between various formulas $\varphi_{a, b}$ and their finite conjunctions. Note that if $a \in B c$ or $d \in b B$ then clearly $\varphi_{a, b}$ implies $\varphi_{c, d}$, and we call such implications trivial. Geometrically these implications correspond to shifts 'right and down' on the plane:

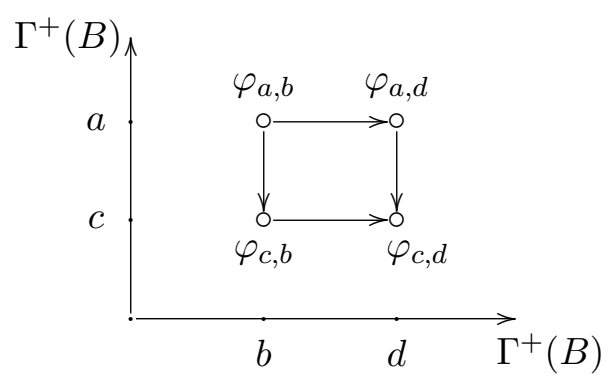

If $B=V$ is a valuation domain, then it follows from [9, L. 12.1] that all implications between pp-formulas $\varphi_{a, b}$, and even between conjuncts of them, are 'trivial', in particular $L(V)$ is freely generated by two chains $\Gamma^{+}(V)$ (and 
in the particular setting of valuation domains it makes sense to represent $\Gamma^{+}(B)=\Gamma^{+}(V)$ as a line, as we did in the previous diagram). As we will see in a moment, the situation for Bézout domains is more complicated: there are some nontrivial implications.

Let us make first a simple remark: if $\varphi, \psi$ are pp-formulas over any ring, then $\varphi$ implies $\psi$ if and only if $\varphi+\psi$ implies $\psi$.

By Lemma 3.1 we have $\varphi_{a, b}+\varphi_{c, d}=\varphi_{\operatorname{gcd}(a, c), \operatorname{lcm}(b, d)}$. Since $\operatorname{gcd}(a, c) \mid c$ and $d \mid \operatorname{lcm}(b, d)$ it follows that, when analyzing the implication $\varphi_{a, b} \rightarrow \varphi_{c, d}$, we may assume that $a \mid c$ and $d \mid b$, that is $c=a g$ and $b=d h$ for some $g, h \in B$.

A similar simplification applies to the (most general) implication $\bigwedge_{i \leq n} \varphi_{a_{i}, b_{i}}$ $\rightarrow \varphi_{c, d}$. Namely, we can replace $\bigwedge_{i \leq n} \varphi_{a_{i}, b_{i}}$ by $\left(\bigwedge_{i \leq n} \varphi_{a_{i}, b_{i}}\right)+\varphi_{c, d}$. By distributivity of the lattice $L(B)$ (see Lemma 2.4) the last formula equals $\bigwedge_{i \leq n}\left(\varphi_{a_{i}, b_{i}}+\varphi_{c, d}\right)$, hence $\bigwedge_{i \leq n} \varphi_{\operatorname{gcd}\left(a_{i}, c\right), \operatorname{lcm}\left(b_{i}, d\right)}$. Thus for each $i \leq n$ we may assume that $c=a_{i} g_{i}$ and $b_{i}=d h_{i}$ for some $g_{i}, h_{i} \in B$.

First we will analyze the case when $d \neq 0$ and $a_{i} \neq 0$ for every $i$. We consider the remaining 'degenerate' cases later.

Proposition 3.2. Let $B$ be a Bézout domain, $a_{i}, g_{i}, h_{i}, i \leq n$ and $c, d$ be elements of $B$ such that $c=a_{i} g_{i}$ for all $i$. Further assume that $a_{i}, d \neq 0$ and $l_{i}=\operatorname{lcm}\left(g_{i}, h_{i}\right)$ for all $i$ again. Then the following are equivalent:

1) $\bigwedge_{i \leq n} \varphi_{a_{i}, d h_{i}}$ implies $\varphi_{c, d}$.

2) $c B+\sum_{i \leq n} g_{i} h_{i} B=B$.

3) $c B+\sum_{i \leq n} l_{i} B=B$.

Proof. 1) $\Rightarrow 2$ ). Otherwise there exists a prime ideal $P$ containing $c B+$ $\sum_{i \leq n} g_{i} h_{i} B$. The implication holds true also over $B_{P}$ which is a valuation domain. It follows from [9, Cor. 12.4] that for some $i$ we have both $a_{i} g_{i} \mid a_{i}$ in $R_{P}$, that is $g_{i} \notin P$, and $d h_{i} \mid d$ in $R_{P}$, that is $h_{i} \notin P$. But then $g_{i} h_{i} \notin P$, a contradiction.

2) $\Rightarrow 3$ ). Since $l_{i}$ divides $g_{i} h_{i}$, this is obvious.

$3) \Rightarrow 1$ ). It suffices to prove that our implication holds true over any localization $B_{P}$. Since $B_{P}$ is a valuation domain, our equality implies that either $c$ is invertible, or one of $l_{i}$ is invertible. If $c$ is invertible, then the formula $\varphi_{c, d}$ holds everywhere, so there is nothing to prove.

Suppose that $l_{i}$ is invertible in $B_{P}$, that is $l_{i} \notin P$. This clearly implies $g_{i}, h_{i} \notin P$, and we obtain the desired arguing as in 1 ). 
Note that our proof of the proposition (as most proofs using localizations) says nothing about how this implication is 'realized' as a sequence of 'trivial' implications (see [7, p. 12] for the meaning of triviality). In fact one can give a completely constructive proof of this claim in the spirit of [11]. However we decided to suppress this longish proof just occasionally producing a string of 'real' implications.

But for future use it is worthwhile to give a 'geometric' interpretation of this result. We slice our $\Gamma^{+}(B) \times \Gamma^{+}(B)$ plane in four quarters $I, I I, I I I, I V$. The following diagram represents these quarters in a neighborhood of the point $\varphi_{c, d}$ corresponding to the elements $c, d \in B$.

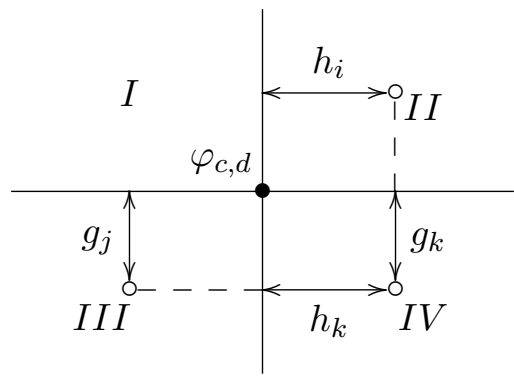

To produce an implication $\bigwedge_{i \leq n} \varphi_{a_{i}, b_{i}} \rightarrow \varphi_{c, d}$ we proceed as follows. After summing up and replacing each given $\varphi_{a_{k}, b_{k}}(k \leq n)$ with $\varphi_{a_{k}, b_{k}}+\varphi_{c, d}$, we will push the formula $\varphi_{a_{k}, b_{k}}$ into the fourth quarter. The result of this operation for formulas in the second and third quarters, so of the form $\varphi_{a_{i}, b_{i}}$ with $c \mid a_{i}$ and $\varphi_{a_{j}, b_{j}}$ with $b_{j} \mid d$ respectively, is shown by dashed lines on the diagram. Now we could collect the products $g_{k} h_{k}$ creating our ideal $J=c B+\sum_{i \leq n} g_{i} h_{i} B$.

If we are persistent enough to get $J=B$, then we obtain the desired implication. For instance this is the case if (different) $g_{j_{1}}$ and $g_{j_{2}}$ in the third quarter are coprime.

When $n=1$, the things are simplified.

Corollary 3.3. Let $a, b, c, d$ be elements of a Bézout domain $B$ with $a, d \neq 0$. Then $\varphi_{a, d h}$ implies $\varphi_{a g, d}$ if and only if $g$ is invertible and $a, h$ are coprime.

Proof. By Lemma 3.2 our implication takes place if and only if $a g B+g h B=$ $B$. This clearly requires that $g$ is invertible, and then that $a$ and $h$ are coprime. 


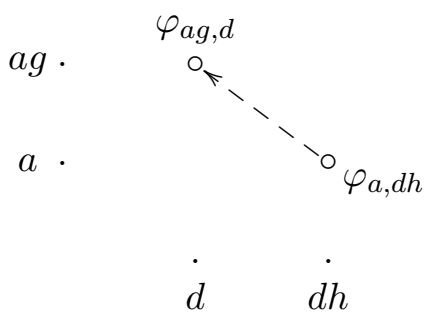

Referring to the previous diagram this means that the (single) formula $\varphi_{a, b}$ that implies $\varphi_{c, d}$ is located in the first and second quarters, therefore lies 'above' $\varphi_{c, d}$.

Warning: in the previous diagram, as well as in the next ones representing the plane $\Gamma^{+}(B) \times \Gamma^{+}(B)$ in the remainder of the paper, we draw axes neither as lines nor as encircled regions and we simply indicate the involved "coordinates" $a, d, \ldots$ on each of them.

The following useful lemma describes implications between single formulas $\varphi_{a, b}$, and is also a direct consequence of Proposition 3.2.

Lemma 3.4. Let $a, b, c, d$ be elements of a Bézout domain $B, a, d \neq 0$. Then $\varphi_{a, b} \rightarrow \varphi_{c, d}$ if and only if $c \mid a$ and elements $c$ and $\operatorname{lcm}(b, d) / d$ are coprime.

It follows from Corollary 3.3 that, if $a$ and $b^{\prime}$ are coprime, then the formulae $\varphi_{a, b}$ and $\varphi_{a, b b^{\prime}}$ are equivalent. This has an obvious consequence for principal ideal domains. More generally a Bézout domain $B$ is said to be adequate, if for all $0 \neq a, b \in B$ we can decompose $a=c d$ such that $d$ and $b$ are coprime, and for any $c^{\prime} \in B$ such that $c^{\prime} \mid c$ and the ideal $c^{\prime} B$ is proper, $c^{\prime}$ and $b$ are not coprime. For instance (see [2, Sec. 3]) the ring of entire complex or real functions in one variable is adequate.

Corollary 3.5. Let $B$ be an adequate Bézout domain. Then every 1-ppformula over $B$ is equivalent to a finite conjunction of pp-formulas $\varphi_{a, b}$, where $a$ and $b$ are coprime.

For the sake of completeness we will also describe the remaining 'degenerate' implications $\bigwedge_{i \leq n} \varphi_{a_{i}, b_{i}} \rightarrow \varphi_{c, d}$. If $d=0$, then the formula $\varphi_{c, d}$ is equivalent to the formula $x=x$, therefore holds everywhere. Thus it remains to consider the case when certain $a_{i}$ equal zero. Note that in this case $c=a_{i} g_{i}=0$, therefore $\varphi_{c, d}$ is just the annihilator formula $x d=0$.

Lemma 3.6. Let $a_{1}, \ldots, a_{n}, b_{1}, \ldots, b_{n}, d$ be elements of a Bézout domain $B$ such that $d \neq 0, a_{1}=\cdots=a_{k}=0, a_{k+1}, \ldots, a_{n} \neq 0$, and $b_{i}=d h_{i}$ for each $i$. Then $\bigwedge_{i \leq n} \varphi_{a_{i}, b_{i}} \rightarrow x d=0$ if and only if $d \in \sum_{i=1}^{k} b_{i} B$. 
Proof. Using elementary duality, our implication can be rewritten as the implication $d \mid x \rightarrow \sum_{i \leq n}\left(b_{i} \mid x \wedge a_{i} x=0\right)$ of left pp-formulae. Note that $(B, d)$ is a free realization of the formula $d \mid x$. Thus this implication takes place if and only if $d \in \sum_{i \leq n}\left(b_{i} \mid x \wedge a_{i} x=0\right)(B)$. If $a_{i} \neq 0$, then the ideal $\left(a_{i} x=0\right)(B)$ equals 0 , therefore we can restrict the range of the sum to $i \leq k$. For $i \leq k$, the ideal $\left(a_{i} x=0\right)(B)$ equals $B$, therefore the whole sum equals $\sum_{i \leq k} B b_{i}$, as desired.

\section{The Ziegler SPeCtrum}

Recall that the Ziegler spectrum, $\mathrm{Zg}_{R}$, of a ring $R$ is a (quasi-compact) topological space whose points are (isomorphism classes of) indecomposable pure injective $R$-modules, and the basis of topology is given by (compact) open sets $(\varphi / \psi)=\left\{M \in \mathrm{Zg}_{R} \mid m \in \varphi(M) \backslash \psi(M)\right.$ for some $\left.m \in M\right\}$, where $\varphi$ and $\psi$ are pp-formulae in one variable. This basic open set is nonempty if and only if $\varphi$ does not imply $\psi$.

The following result is an standard consequence of Lemma 2.3.

Corollary 4.1. Let $B$ be a Bézout domain. Then the basis for $\mathrm{Zg}_{B}$ is given by open sets $\left(\psi_{c, d} / \varphi_{a, b}\right)$, that is $(c \mid x \wedge x d=0) /(a \mid x+x b=0)$, where $a, b, c, d \in B$.

Of course it is useful to know when such an open set is nontrivial. We will give an answer only in the most interesting 'non-degenerate' case. The remaining cases are analyzed without difficulty.

Lemma 4.2. Let $a, b, g, h$ be nonzero elements of a Bézout domain $B$. Then the implication $a|x \wedge x b h=0 \rightarrow a g| x+x b=0$ holds if and only if $g$ and $h$ are coprime.

Proof. To analyze this implication, arguing as usual, we have to replace the formula $a \mid x$ by $a \mid x+\varphi_{a g, b} \doteq \varphi_{a, b}$. Thus $g_{1}=g$ and $h_{1}=1$, hence $g_{1} h_{1}=g$ in the notation of Proposition 3.2. Similarly we will replace the formula $x b h=0$ by $x b h=0+\varphi_{a g, b} \doteq \varphi_{a g, b h}$, therefore $g_{2}=1$ and $h_{2}=h$, hence $g_{2} h_{2}=h$.

By the same proposition our implication holds if and only if $a g B+g B+$ $h B=B$, that is if $g$ and $h$ are coprime.

For instance we see that there could be nontrivial 'relations' when generating $L(B)$ by two copies of $\Gamma^{+}(B)$, that is $L(B)$ is usually a proper factor of the (distributive) lattice freely generated by these copies. 
An algebraic description of points of $\mathrm{Zg}_{B}$ is in principle known from Ziegler [14, p. 168, Remark]. Namely, every such point (that is, an indecomposable pure injective module) $M$ localizes: there is a prime ideal $P$ such that every element not in $P$ acts as an isomorphism on $M$. Thus $M$ is an indecomposable pure injective module over the valuation domain $B_{P}$, therefore (see [14] again) is isomorphic to the pure injective envelope of the factor $I / J$, where $J \subset I$ are fractional ideals of $B_{P}$.

However, as for valuation domains, a syntactical description of points of $\mathrm{Zg}_{B}$ through pp-types has many advantages. Recall that a (non-overlapping) collection $p=p^{+} \cup \neg p^{-}$of pp-formulas and their negations is called a $p p$ type, if it is 1) complete, that is any pp-formula belongs to $p^{+}$or $p^{-}$, and 2) consistent, that is if $\varphi_{1}, \ldots, \varphi_{n} \in p^{+}, \varphi$ is a pp-formula and $\bigwedge_{i \leq n} \varphi_{i} \rightarrow \varphi$, then $\varphi \in p^{+}$. Because each pp-type $p$ is uniquely determined by its positive part $p^{+}$, we usually identify $p$ with $p^{+}$(and write, for a pp-formula $\varphi, \varphi \in p$ for $\varphi \in p^{+}$and $\varphi \notin p$ for $\varphi \in p^{-}$).

A general theory (see $[7$, Ch. 4] says that for any pp-type $p$ there is a (minimal) unique pure injective pointed module $(N(p), m)$, the pure injective envelope of $p$, such that $p$ coincides with the pp-type of $m$ in $N(p)$, where $p p_{N(p)}(m)=\{\varphi \mid N(p) \models \varphi(m)\}$. (Actually a similar notion of pp-type makes sense for any element $m$ in any module $M$.) A pp-type $p$ is said to be indecomposable, if the module $N(p)$ is indecomposable. Thus to describe points of $\mathrm{Zg}_{B}$ is the same as to describe 1) indecomposable pp-types as subsets of $L(B)$ and 2 ) give a criterion when different pp-types will produce isomorphic indecomposable pure injective modules.

Now we are in a position to realize this program for Bézout domains. Since the lattice $L(B)$ is distributive, from [14, Thm. 4.4] we obtain the following criterion of indecomposability.

Fact 4.3. A pp-type $p$ over a Bézout domain is indecomposable if and only if for all $\varphi, \psi \in p^{-}$we have $\varphi+\psi \in p^{-}$.

Recall that a nonempty subset $F$ of a lattice is said to be a filter, if it is upward closed (that is $a \in F$ and $a \leq b$ implies $b \in F$ ), and closed with respect to the meet (that is $a, b \in F$ implies $a \wedge b \in F$ ). Since every pptype $p$ is closed with respect to conjunctions, it is always a filter in $L(B)$. The above lemma shows that indecomposable pp-types over Bézout domains correspond to filter-cofilter partitions of $L(B)$, where a cofilter is a nonempty subset of a lattice which is downward closed and closed with respect to join. 
But we could essentially improve this 'rough' description for Bézout domains.

Let $p$ be an indecomposable pp-type over a Bézout domain $B$. We could restrict the above partition of $L(B)$ to divisibility and annihilator conditions. Namely, we set $I(p)=\left\{b \in \Gamma^{+}(B): x b=0 \in p\right\}, I^{*}(p)=\Gamma^{+}(B) \backslash I(p)$ and $J(p)=\left\{a \in \Gamma^{+}(B): a \mid x \notin p\right\}, J^{*}(p)=\Gamma^{+}(B) \backslash J(p)$.

Lemma 4.4. Let $p$ be an indecomposable pp-type over a Bézout domain B and $I=I(p), J=J(p)$. Then

1) $\Gamma^{+}(B)=I \cup I^{*}$ is a filter-cofilter partition of $\Gamma^{+}(B)$.

2) $\Gamma^{+}(B)=J \cup J^{*}$ is a filter-cofilter partition of $\Gamma^{+}(B)$.

3) If $a<c$ for $a \in J^{*}, c \in J$ and $d<b$ for $b \in I, d \in I^{*}$ then the elements $g=c / a$ and $h=b / d$ are not coprime.

Proof. 1) If $b \in I$ and $b \leq b^{\prime}$, then $x b=0$ implies $x b^{\prime}=0$, therefore $b^{\prime} \in I$, hence $I$ is upward closed. The fact that $I$ is closed with respect to gcd follows from Lemma 3.14 ). Similarly Fact 4.3 and Lemma 3.13 ) imply that $I^{*}$ is a cofilter.

2) has a similar proof (but now the filter if $J^{*}$ ).

3) Suppose that $g$ and $h$ are coprime. We have that $a \mid x, x b=0 \in p$ and $c \mid x, x d=0 \in p^{-}$. By Lemma 4.2 we obtain $a|x \wedge x b=0 \rightarrow c| x+x d=0$, therefore the last formula is in $p$. But, since $p$ is indecomposable, Fact 4.3 implies that either $c \mid x \in p$, hence $c \in J^{*}$, or $x d=0 \in p$, hence $d \in I$, a contradiction in both cases.

Note that the pair of filter-cofilter partitions in this lemma is uniquely determined by the filter $I$ and cofilter $J$. We call a pair $(I, J)$ satisfying the conditions of this lemma admissible, and we will usually write this pair instead of the two corresponding partitions of $\Gamma^{+}(B)$.

We will show that Lemma 4.4 characterizes indecomposable pp-types over Bézout domains. But first let us clarify a little the objects that occur in this lemma. Note that the filters in $\Gamma^{+}(B)$ correspond one-to-one to ideals of $B$, via the map $F \mapsto I(F) \doteq\{b \in B \mid b B \in F\}$. For instance it is easily seen that $I(F)$ is an ideal, in fact

- $I(F)$ includes 0 ,

- if $a \in I(F)$ then $a \leq a r$ implies $a r \in I(F)$ for any $r \in B$,

- $I(F)$ is closed under gcd and consequently under + .

If $P$ is a prime ideal of $B$ and $F=F(P)=\{b B \mid b \in P\}$ is the corresponding filter, then $F^{*}=\Gamma^{+}(B) \backslash F$ is clearly a cofilter. Unfortunately in 
general we cannot claim that for a filter-cofilter partition $\Gamma^{+}(B)=F \cup F^{*}$ the ideal $I=I(F)$ is prime. What we have is apparently weaker: $a, b \notin I$ implies $\operatorname{lcm}(a, b) \notin I$ (but $a b \in I$ is quite possible).

Now we give the promised classification of indecomposable pp-types.

Theorem 4.5. Let $B$ be a Bézout domain. Then there exists a natural oneto-one correspondence between indecomposable pp-types in one variable over $B$ and admissible pairs $(I, J)$ of filter-cofilter partitions of $\Gamma^{+}(B)$.

Proof. Given two filter-cofilter partitions $(I, J)$ as above, we will construct an indecomposable pp-type $p=p(I, J)$ as follows. Take a divisibility formula $a \mid x$ in $p$ if $a \in J^{*}$, and take it in $p^{-}$otherwise. Now include an annihilator formula $x b=0$ in $p$ if $b \in I$, and put in into $p^{-}$otherwise.

Further include a formula $\varphi_{a, b}$ in $p$ if $a \mid x$ is already in $p$ or $x b=0 \in p$ (and take $\varphi_{a, b}$ in $p^{-}$otherwise). Finally put the formula $\bigwedge_{i \leq n} \varphi_{a_{i}, b_{i}}$ in $p$ if and only if each $\varphi_{a_{i}, b_{i}}$ is already in $p$.

From Lemma 2.3 it follows that $p$ is complete, and Fact 4.3 would yield indecomposability. What remains is to prove the consistency of $p$. Suppose, by a way of contradiction, that $\varphi_{1}, \ldots, \varphi_{n} \in p$ and $\bigwedge_{i \leq n} \varphi_{i} \rightarrow \varphi$ with $\varphi \in p^{-}$. By the definition of $p$ we may assume that each $\varphi_{i}$ is either a divisibility or an annihilator formula. Furthermore, since $J^{*}$ and $I$ are filters, we could further suppose that just one of those formulas occurs, that is the whole implication is of the form $a \mid x \wedge x b=0 \rightarrow \varphi, a \in J^{*}, b \in I$. Furthermore clearly we may assume that $\varphi \doteq \varphi_{c, d} \in p^{-}$, therefore $c \in J$ and $d \in I^{*}$.

Finally taking the sums we can replace in this implication $a$ with $\operatorname{gcd}(a, c) \in$ $J^{*}$ and $b$ with $\operatorname{lcm}(b, d) \in I$. But then $a<c$ and $d<b$, therefore elements $g=c / a$ and $h=b / d$ are coprime by Lemma 4.2. But this contradicts admissibility (see condition 3) of Lemma 4.4).

Thus we have constructed by each indecomposable pp-type a pair of filtercofilter partitions of $L(B)$ satisfying 3 ) and vice versa. The proof that these maps invert each other is straightforward.

In general the hypotheses of this theorem are quite enigmatic and, over a particular ring, may require a further nontrivial analysis. Let us see what they mean in the case when $B$ is a principal ideal domain. Then the filter $I$ corresponds to a principal ideal $r B, r \in B$. If $r \neq 0$ then the condition that $I^{*}$ is cofilter clearly implies that $r=p^{n}$ for an irreducible $p \in B$. Similarly we obtain that either $J=0$ or $J=q^{m} B$ for an irreducible $q \in B$. Finally if $I=p^{n} B$ and $J=q^{m} B$ then 3 ) yields $p=q$. This way we clearly obtain 
the (well known — see [7, Sec. 5.2.1]) classification of indecomposable pure injective modules over principal ideal domains. For instance, if $B=\mathbb{Z}$ and $I=4 \mathbb{Z}, J=8 \mathbb{Z}$, then we obtain the finite abelian group $\mathbb{Z} / 16 \mathbb{Z}$; and for $I=4 \mathbb{Z}, J=0$ we get the 2-Prüfer group $\mathbb{Z}_{2_{\infty}}$.

Thus we have classified indecomposable pp-types. To complete a (syntactical) classification of points of $\mathrm{Zg}_{B}$ we have to describe which pp-types produce isomorphic indecomposable pure injective modules (that is their pure injective envelopes are isomorphic). First we need a technical lemma describing how the multiplication by an element of $B$ changes the pp-type of an element of an indecomposable pure injective $B$-module.

Lemma 4.6. Let $m$ be an element of an indecomposable pure injective module $M$ with the pp-type $p$, and let $(I, J)$ be the corresponding admissible pair. Further assume that $b \notin I$ (that is $m b \neq 0$ in $M$ ) and let $\left(I^{\prime}, J^{\prime}\right)$ be the admissible pair corresponding to the pp-type $q$ of $m b$ in $M$. Then $I^{\prime}=(I$ : $b)=\{r \in B \mid b r \in I\}$ and $J^{* *} \doteq J_{b}=\left\{c \in B \mid c^{\prime}=c / \operatorname{gcd}(c, b) \in J^{*}\right\}$.

Proof. For $r \in B$ we have $r \in I^{\prime}$ (that is $x r=0 \in q$ ) if and only if $(m b) r=0$ in $M$, that is $b r \in I$. Thus $I^{\prime}=(I: b)$.

Now we would like to calculate $J^{\prime *}$. By the definition, $c \in J^{\prime *}$ if and only if $c$ divides $m b$ in $M$, that is the formula $c \mid x b$ belongs to $p$. Let $d=\operatorname{gcd}(c, b)$ and $c^{\prime}=c / d$. We claim that this happens exactly when $c^{\prime} \in J^{*}$.

Firstly, by the proof of Lemma 2.3, the formula $c \mid x b$ is equivalent to $c^{\prime} \mid x+x d=0$.

Note also that $b \in d B$ and $m b \neq 0$ implies $m d \neq 0$ in $M$, that is $x d=$ $0 \in p^{-}$. Since $p$ is indecomposable, it follows that the formula $c\left|x d \doteq c^{\prime}\right|$ $x+x d=0$ is in $p$ if and only if $c^{\prime} \mid x \in p$, that is $c^{\prime} \in J^{*}$.

For instance we conclude from this lemma (in fact this is evident) that $J^{*} b \subseteq J^{*}$. But also (less trivially) if $c$ and $b$ are coprime then $c \in J^{*}$ if and only if $c \in J^{\prime *}$.

Below by $p(I, J)$ we will denote (the unique) indecomposable pp-type corresponding to the admissible pair $(I, J)$, and by $N(I, J)$ we will denote the pure injective envelope of this type.

Informally the following result says that the types of two nonzero elements in an indecomposable pure injective $B$-module can be equalized when multiplying by an element of $B$. This lemma completes the classification of $\mathrm{Zg}_{B}$ 
Lemma 4.7. Let $\left(I_{1}, J_{1}\right)$ and $\left(I_{2}, J_{2}\right)$ be admissible filter-cofilter partitions of $\Gamma^{+}(B)$. Then the corresponding indecomposable pure injective modules are isomorphic if and only if one of the following holds:

1) there exists $b \notin I_{1}$ such that $I_{2}=\left(I_{1}: b\right)$ and $J_{2}=\left(J_{1}\right)_{b}$, or

2) there exists $d \notin I_{2}$ such that $I_{1}=\left(I_{2}: d\right)$ and $J_{1}=\left(J_{2}\right)_{d}$.

Proof. $\Leftarrow$. By symmetry we may assume 1$)$. Let $M=N\left(I_{1}, J_{1}\right)$ be an indecomposable pure injective module with an element $m \in M$ whose pptype equals $p\left(I_{1}, J_{1}\right)$. Then $m b \neq 0$, and (by Lemma 4.6) the pp-type of $m b$ in $M$ equals $p\left(I_{2}, J_{2}\right)$. Since $M$ is indecomposable, it follows that $N\left(I_{2}, J_{2}\right) \cong M$.

$\Rightarrow$. Let $M=N\left(I_{1}, J_{1}\right)$ be as above. By the assumption, there exists $n \in M$ whose pp-type equals $p\left(I_{2}, J_{2}\right)$. We have already mentioned that $M$ is localized: there exists a prime ideal $P$ of $B$ such that $M$ is an indecomposable pure injective module over the valuation domain $B_{P}$ (that is every $r \in B \backslash P$ acts as an isomorphism of $M$ ).

It follows from [9, L. 11.11] that there exist elements $b^{\prime}, d^{\prime} \in B_{P}$ such that either $p p_{M}\left(m b^{\prime}\right)=p p_{M}(n)$ or $p p_{M}(m)=p p_{M}\left(n d^{\prime}\right)$, and we consider only the first possibility. Let $b^{\prime}=b r^{-1}$ for some $b \in B$ and $r \notin P$. Then $k=m b$ realizes in $M$ the same pp-type as $n$, that is $p\left(I_{2}, J_{2}\right)$. Thus by Lemma 4.6 we obtain $I_{2}=\left(I_{1}: b\right)$ and $J_{2}=\left(J_{1}\right)_{b}$.

\section{TYPES}

In this section we will give a syntactical description of pp-types in one variable over Bézout domains $B$. Recall that we view $\Gamma^{+}=\Gamma^{+}(B)$ as the factor set of $B$ modulo the set of units of $B$, or also as the set of principal ideals of $B$, but for simplicity we sometimes identify the elements of $B$ with the corresponding equivalence classes, or principal ideals. Then $\Gamma^{+}$is a lattice with the ordering $a \leq b$ if $b \in a B$, that is $a B \supseteq b B$. For instance 1 (more precisely $B=1 B$ ) is the smallest element of this ordering and 0 (that is, $0 B$ ) is the largest element. Recall that a cofilter of the lattice $\Gamma^{+}$is a nonempty subset of $\Gamma^{+}$which is downward closed and closed with respect to lcm: 


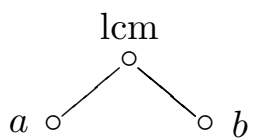

By $\widehat{\Gamma}^{+}$we denote the set of cofilters of $\Gamma^{+}$with respect to inclusion relation $\subseteq$. It is not difficult to check that $\widehat{\Gamma}^{+}$is a lattice, where the greatest lower bound is given by intersection. The least upper bound of two cofilters $\Lambda$ and $\Lambda^{\prime}$ consists of elements $a$ such that $a \leq \operatorname{lcm}\left(c, c^{\prime}\right)$ for some $c \in \Lambda, c^{\prime} \in \Lambda^{\prime}$ :

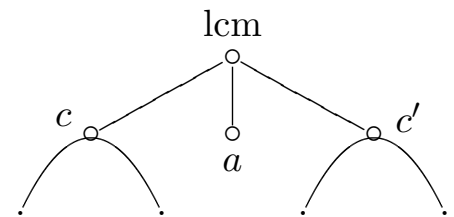

We will denote this least upper bound by $\left\langle\Lambda \cup \Lambda^{\prime}\right\rangle$, in fact it is the minimal cofilter extending $\Lambda \cup \Lambda^{\prime}$.

Note that $\Gamma^{+}$itself, considered as a set of principal ideals, is naturally contained in $\widehat{\Gamma}^{+}$, indeed $\widehat{\Gamma}^{+}$is its completion.

Recall that a pp-type $p$ is just a filter on $L(B)$. It follows from Lemma 2.3, that $p$ is uniquely determined by the set of formulas $\varphi_{a, b} \in p^{+}$. We associate to $p$ a function $F=F(p): \Gamma^{+} \rightarrow \widehat{\Gamma}^{+}$given by $F(b)=\left\{a \in B \mid \varphi_{a, b} \in p\right\}$ for all $b \in B$ (viewed as an element of $\Gamma^{+}$).

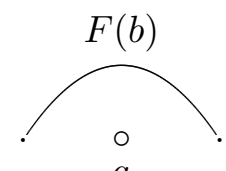

Notice that, if $a, a,^{\prime}, b, b^{\prime} \in B$ are such that $a B=a B^{\prime}$ and $b B=b^{\prime} B$, then the formulas $\varphi_{a, b}$ and $\varphi_{a^{\prime}, b^{\prime}}$ are equivalent, therefore our definition is sound.

Lemma 5.1. $F(b)$ is a cofilter for any $b \in B$.

Proof. First of all $F(b)$ is nonempty. Namely $\varphi_{1, b}$ is equivalent to $x=x$, therefore belongs to $p$, hence $1 \in F(b)$.

Furthermore, $F(b)$ is downward closed. Indeed if $\varphi_{a, b} \in p$ and $a^{\prime} \leq a$, then $\varphi_{a, b} \rightarrow \varphi_{a^{\prime}, b}$ (a trivial implication), therefore $a^{\prime} \in F(b)$.

Finally $F(b)$ is closed with respect to $1 \mathrm{~cm}$. Namely let $a, a^{\prime} \in F(b)$, that is $\varphi_{a, b}, \varphi_{a^{\prime}, b} \in p$. From Lemma 3.1 it easily derives that $\varphi_{\operatorname{lcm}\left(a, a^{\prime}\right), b} \in p$, therefore $\operatorname{lcm}\left(a, a^{\prime}\right) \in F(b)$ 
Let us point out some crucial properties of $F$.

Proposition 5.2. Let $B$ be a Bézout domain, $p$ be a pp-type in one variable $x$ over $B$, and the function $F=F(p): \Gamma^{+} \rightarrow \widehat{\Gamma}^{+}$be defined as before. Then the following holds:

1) $F(0)=\Gamma^{+}$;

2) $F(1)=\Gamma^{+}$if and only if $p$ is zero;

3) for every $a, b, b^{\prime} \in B$ from $a \in F(b)$ it follows that $a b^{\prime} \in F\left(b b^{\prime}\right)$, in particular $F$ is non-decreasing;

4) for every $a, b, b^{\prime} \in B$, if $a \in F\left(b b^{\prime}\right)$ and $a$ is coprime with $b^{\prime}$, then $a \in F(b)$;

5) $F$ preserves the lattice meet operation, that is, for every $b, b^{\prime} \in B$ we have $F\left(\operatorname{gcd}\left(b, b^{\prime}\right)\right)=F(b) \cap F\left(b^{\prime}\right)$.

Proof. 1) If $b=0$, then the formula $\varphi_{a, b}$ holds everywhere, therefore $a \in$ $F(b)$.

2) Note that $0 \in F(1)$ if and only if $\varphi_{0,1} \doteq 0 \mid x$ is in $p$.

3) Clearly $\varphi_{a, b}$ implies $\varphi_{a b^{\prime}, b b^{\prime}}$, therefore $a \in F(b)$ yields $a b^{\prime} \in F\left(b b^{\prime}\right)$.

In particular, if $b \leq c$, then $c=b b^{\prime}$ for some $b^{\prime} \in B$. Therefore $a \in F(b)$ implies $a b^{\prime} \in F(c)$, hence $a \in F(c)$. Thus the function $F$ is non-decreasing.

4) follows from a remark after Corollary 3.3.

5) Let $a \in B$ be such that $a \in F\left(\operatorname{gcd}\left(b, b^{\prime}\right)\right)$. Then $a \operatorname{gcd}\left(b, b^{\prime}\right) \mid x \operatorname{gcd}\left(b, b^{\prime}\right)$ is in $p$, therefore (by trivial implications) $\varphi_{a, b}, \varphi_{a, b^{\prime}} \in p$, hence $a \in F(b) \cap$ $F\left(b^{\prime}\right)$.

Conversely, suppose that $a \in F(b) \cap F\left(b^{\prime}\right)$, that is $\varphi_{a, b}, \varphi_{a, b^{\prime}} \in p$. By Lemma 3.1 it follows that $\varphi_{a, b} \wedge \varphi_{a, b^{\prime}}=\varphi_{a, \operatorname{gcd}\left(b, b^{\prime}\right)} \in p$, hence $a \in F\left(\operatorname{gcd}\left(b, b^{\prime}\right)\right)$.

We have just seen that the function $F$ preserves the meet. Now we investigate the behavior of $F$ with respect to the join.

Lemma 5.3. Let $F=F(p): \Gamma^{+} \rightarrow \widehat{\Gamma}^{+}$be defined as before and $b, b^{\prime} \in B$. Then $F\left(\operatorname{lcm}\left(b, b^{\prime}\right)\right) \supseteq\left\langle F(b) \cup F\left(b^{\prime}\right)\right\rangle$.

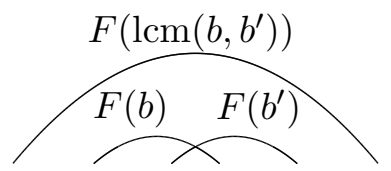


Proof. By symmetry it suffices to check that $F(b) \subseteq F\left(\operatorname{lcm}\left(b, b^{\prime}\right)\right)$. Let $a \in F(b)$, that is $\varphi_{a, b} \in p$. But the last formula trivially implies $\varphi_{a, \operatorname{lcm}\left(b, b^{\prime}\right)}$, therefore $\varphi_{a, \operatorname{lcm}\left(b, b^{\prime}\right)} \in p$ yields $a \in F\left(\operatorname{lcm}\left(b, b^{\prime}\right)\right)$

The opposite inclusion $F\left(\operatorname{lcm}\left(b, b^{\prime}\right)\right) \subseteq\left\langle F(b) \cup F\left(b^{\prime}\right)\right\rangle$ does not hold in general. In fact it is easily seen that our claim is equivalent to the following. If $a \in B$ and $\varphi_{a, \operatorname{lcm}\left(b, b^{\prime}\right)} \in p$, then there are $c, c^{\prime} \in B$ such that $a \mid \operatorname{lcm}\left(c, c^{\prime}\right)$ and $\varphi_{c, b}, \varphi_{c^{\prime}, b^{\prime}}$ are in $p$.

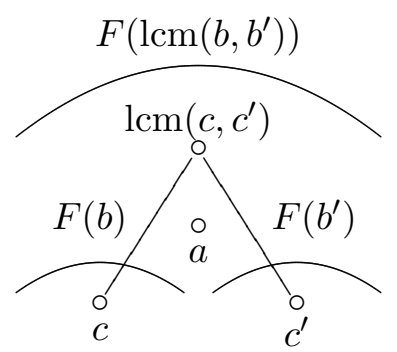

However let $B=\mathbb{Z}$ and let $p$ be the pp-type of the element $m=(2,1)$ in the $\mathbb{Z}$-module $\mathbb{Z} \oplus \mathbb{Z}_{12}$. Take $a=2, b=4$ and $b^{\prime}=6$, whence $\operatorname{lcm}\left(b, b^{\prime}\right)=12$. Then $m$ satisfies $\varphi_{a, \operatorname{lcm}\left(b, b^{\prime}\right)}$, that is $\varphi_{2,12} \doteq 24 \mid 12 x$, therefore $2 \in F(12)$. On the other hand $2 \notin\langle F(4) \cup F(6)\rangle$. Indeed otherwise there are $c, c^{\prime} \in \mathbb{Z}$ such that $2 \mid \operatorname{lcm}\left(c, c^{\prime}\right)$ and $\varphi_{c, b}, \varphi_{c^{\prime}, b^{\prime}} \in p$. These conditions mean $4 c \mid 4(2,1)$ and $6 c \mid 6(2,1)$. This clearly implies $c, c^{\prime}= \pm 1$, hence $\operatorname{lcm}\left(c, c^{\prime}\right)=1-\mathrm{a}$ contradiction, since $\operatorname{lcm}\left(c, c^{\prime}\right)$ is even.

Let now $F$ be a function from $\Gamma^{+}$to $\widehat{\Gamma}^{+}$satisfying the conditions 1)-5) of Lemma 5.2. Define a pp-type $p=p(F)$ as follows. Take $\varphi_{a, b} \in p^{+}$if $a \in F(b)$, and take $\varphi_{a, b} \in p^{-}$otherwise. It follows from Lemma 2.3 that, if this set of formulae is consistent, it it uniquely extended to a pp-type over $B$. We claim that conditions 1)-5) give also a sufficient condition for that.

Theorem 5.4. Let $B$ be a Bézout domain. Then there is a natural one to one correspondence between nonzero pp-types $p$ in one variable over $B$ and functions $F: \Gamma^{+} \rightarrow \widehat{\Gamma}^{+}$satisfying the conditions 1)-5) of Lemma 5.2.

Proof. By Lemma 5.2 and what we have already noticed, it suffices to prove that the type $p$ defined by a function $F$ is consistent.

Furthermore (by compactness and taking direct products) it is enough to show that the implication $\bigwedge_{i \leq n} \varphi_{a_{i}, b_{i}} \rightarrow \varphi_{c, d}$ for $\varphi_{a_{i}, b_{i}} \in p$, that is $a_{i} \in F\left(b_{i}\right)$, yields $\varphi_{c, d} \in p$, that is $c \in F(d)$.

As usual (see arguments before Proposition 3.6) we may assume that, for every $i, c=a_{i} g_{i}$ and $b_{i}=d h_{i}$ for some $g_{i}, h_{i} \in B$. 
First suppose that $a_{i} \neq 0$ for every $i$, and also $d \neq 0$. From $a_{i} \in F\left(b_{i}\right)=$ $F\left(d h_{i}\right)$ and $c=a_{i} g_{i}$ by 3$)$ we conclude that $c \in F\left(d g_{i} h_{i}\right)$ for every $i$. Then $c \in \cap_{i \leq n} F\left(d g_{i} h_{i}\right)$, which by 5$)$ equals $F\left(\operatorname{gcd}\left(d g_{i} h_{i}\right)\right)=F\left(d \operatorname{gcd}\left(g_{i} h_{i}\right)\right)$. On the other hand, by Proposition $3.2 c$ and $\operatorname{gcd}\left(g_{i} h_{i}\right)$ are coprime. Then we can apply 4) to conclude $c \in F(d)$.

The case $d=0$ is trivial, because $F(0)=\Gamma^{+}$.

Finally suppose that $d \neq 0$ and $a_{i}=0$ for some $i \leq n$, hence $c=0$. In the notation of Lemma 3.6 this boils down to analyzing the implication $\bigwedge_{i=1}^{k} x b_{i}=0 \rightarrow x d=0$, where $\bigwedge_{i=1}^{k} x b_{i}=0 \in p$ and $d \in \sum_{i \leq k} b_{i} B$. Thus we have $0 \in F\left(b_{i}\right)$ for every $i \leq k$, therefore $0 \in \cap_{i \leq k} F\left(b_{i}\right)=F\left(\operatorname{gcd}\left(b_{1}, \ldots, b_{k}\right)\right)$. From $d \in \sum_{i \leq k} b_{i} B$ we obtain $d \geq \operatorname{gcd}\left(b_{1}, \ldots, b_{k}\right)$, therefore $0 \in F(d)$ since $F$ is nondecreasing. But then $x d=0 \in p$.

\section{Superdecomposable pure injective modules.}

A (non-zero) module $M$ is said to be superdecomposable, if it contains no indecomposable direct summands. If $m$ is a nonzero element of $M$ and $p=p p_{M}(m)$ then, being a direct summand of $M$, the module $N(p)$ is also superdecomposable. Thus a ring $R$ possesses a superdecomposable pure injective module if and if there exists a superdecomposable pp-type in one variable over $R$. A useful necessary condition of the existence of such a type is given in terms of width of the lattice of pp-formulae.

Let $L$ be a lattice with minimum and maximum. For a definition of width of $L$ see [14, p. 182]. For instance $w(L)=0$ if $L$ is a chain, that is all elements of $L$ are comparable. Furthermore, for an ordinal $\alpha$, we (inductively) have $w(L) \geq \alpha+1$ if there are incomparable $a, b \in L$ such that both intervals $[a \wedge b, a]$ and $[a \wedge b, b]$ have width $\geq \alpha(\wedge$ and $\vee$ denote here the meet and join operations of $L$, respectively).

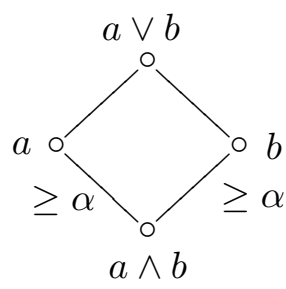

If $w(L) \geq \alpha$ for every $\alpha$, then we say that the width of $L$ is undefined, or that $L$ has no width, and write $w(L)=\infty$. In particular, $w(L)$ is undefined if $L$ contains a countable squeezing family of incomparable diamonds (below we show just the beginning of this sequence): 


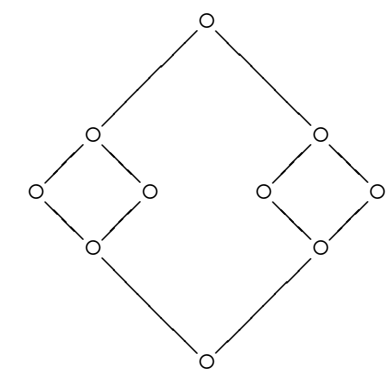

For more tricky "if and only if condition" for non-existence of width in terms of wide subposets see [7, Thm. 7.3.1].

Fact 6.1. [14, Thm. 7.1] If there exists a superdecomposable pure injective module over a ring $R$, then the lattice $L(R)$ has no width. Furthermore, if the lattice $L(R)$ is countable (in particular if $R$ is countable), then the converse is true.

For uncountable rings, the sufficiency of this width condition (to the existence of a superdecomposable pure injective module) is an open problem (see some discussion in [7, Sec. 7.3]).

Before approaching the general case let us first give a useful sufficient condition for the existence of such a module over Bézout domains.

Proposition 6.2. Let $B$ be a Bézout domain with a nonzero proper idempotent ideal $I$ (that is $I=I^{2}$ ). Then $B$ possesses a superdecomposable pure injective module.

Proof. Choose a maximal ideal $P$ containing $I$ and localize at $P$. Then $B_{P}$ is a commutative valuation domain whose (nonzero proper) ideal $I_{P}$ is idempotent. It follows from [9, Thm. 12.12] that there exists a superdecomposable pure injective $B_{P}$-module $M$. This $M$ remains superdecomposable and pure injective when considered as $B$-module.

This situation occurs for Bézout domains quite often.

Example 6.3. The following Bézout domains possess superdecomposable pure injective modules:

1) the ring $A$ of algebraic integers (the algebraic closure of $\mathbb{Q}$ in $\mathbb{C}$ );

2) the ring of entire (complex or real) functions in one variable;

3) the direct product $\mathbb{Z}^{\omega}$ of $\omega$ copies of integers;

4) any filtered product $\mathbb{Z}^{\omega} / F$, where $F$ is a non-principal filter on subsets of $\omega$. 
Proof. We have already mentioned that rings in 1) and 2) are Bézout. Furthermore, the class of Bézout rings is closed with respect to direct products and homomorphic images, therefore the same is true for rings in 3) and 4). In any case we will construct an idempotent ideal $I$ in $B$, and apply Proposition 6.2.

1) Let $I$ be the ideal of $A$ generated by all roots $a_{n}=\sqrt[2^{n}]{2}, n=1, \ldots$. Clearly this ideal is idempotent and proper.

2) Let $c=\left\{c_{n}\right\}$ be a sequence of natural numbers such that $c_{n} \leq n$ for every $n$ and $c_{n} \rightarrow \infty$ (as $n$ goes to $\infty$ ). Clearly the complex function $f_{c}$, defined by $f_{c}(z)=\prod_{n=1}^{\infty}\left(1-z / n^{3}\right)^{c_{n}}$ for all $z$, is entire. Let $I$ be the ideal of the ring $E(\mathbb{C})$ of all entire functions in one variable, generated by such functions.

Note that $f_{c}$ is clearly a multiple of $f_{c^{\prime}}^{2}$, where $c^{\prime}=\left\{c_{n}^{\prime}\right\}$ and, for every $n$, $c_{n}^{\prime}$ is the integer part of $c_{n} / 2$. It follows that $I$ is idempotent.

The same arguments apply to the ring $E(\mathbb{R})$ of analytic real functions in one variable.

3) Consider the ideal $I$ generated by all sequences $\left\{c_{n}\right\}$ as in 2).

4) Consider the image of the ideal $I$ from 3 ).

Note that there exists a visible difference in a behavior of some Bézout domains with regard to existence of superdecomposable pure injective modules. For instance, there is no such module over $\mathbb{Z}$. However, taking a non-principal ultrafilter we see that there exists an elementary equivalent to $\mathbb{Z}$ (in the language of rings) Bézout domain $B$ possessing such a module. Furthermore (by elementary descent) we can easily find a countable model of the theory of $\mathbb{Z}$ with a superdecomposable pure injective module.

But, if $A$ is the ring of algebraic integers, then every element of $A$ is a square. It follows that any model of the theory of $A$ contains a proper idempotent ideal, hence has a superdecomposable pure injective module.

\section{THE NON-EXISTENCE OF WIDTH}

The aim of the remaining part of the paper is to characterize Bézout domains without width. We say that a subset $S$ of a lattice $L$ is dense, if $S$ contains two comparable elements, and for every $a<b \in S$ there exists $c \in S$ such that $a<c<b$. Clearly a lattice $L$ contains a dense subchain if and only if $L$ contains a (countable) subset isomorphic to the ordering of the rationals $(\mathbb{Q}, \leq)$. 
Theorem 7.1. Let $B$ be a Bézout domain. Then the lattice $L(B)$ of ppformulae over $B$ has no width if and only if its value group $\Gamma(B)$ contains a densely ordered subchain.

First we will prove the sufficiency. Let $B$ be a Bézout domain whose value group $\Gamma(B)$ contains a densely ordered countable subchain. It clearly follows that there is a non-unit $g \in B$ that could be 'splitted' infinitely many times: $g=g_{0} \cdot g_{1}$ for nonunits $g_{0}, g_{1} \in B ; g_{0}=g_{00} \cdot g_{01}$ with similar properties, $g_{1}=g_{10} \cdot g_{11}$ and so on.

More formally, there exists a binary tree of nonunits $b_{\eta} \in B, \eta \in 2^{<\omega}$, such that $b_{\eta}=b_{\eta 0} \cdot b_{\eta 1}$. Note that the principal ideals generated by elements located on the same branch of this tree are comparable. But it is quite possible that elements from different branches produce comaximal ideals, for instance $b_{\eta 0}$ and $b_{\eta 1}$ could be coprime.

Note that the formula $\varphi_{g^{2}, g}$ (trivially) implies $\varphi_{g, g^{2}}$ and (by Lemma 3.4) the converse implication does not hold, therefore we obtain a nontrivial interval $\left[\varphi_{g^{2}, g}, \varphi_{g, g^{2}}\right]$ in the lattice $L(B)$ :

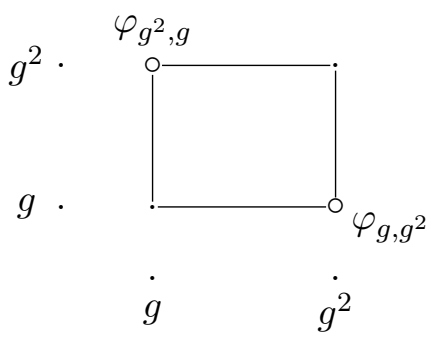

It would be oversimplistic to think of this interval as a square: by Lemma 3.4, all formulas in it are located inside the corresponding horizontal strip, but could protrude outside the square. However we will prove that this interval does not have width, and all the required calculations will be carried through inside this square.

Consider the first step of our construction. 


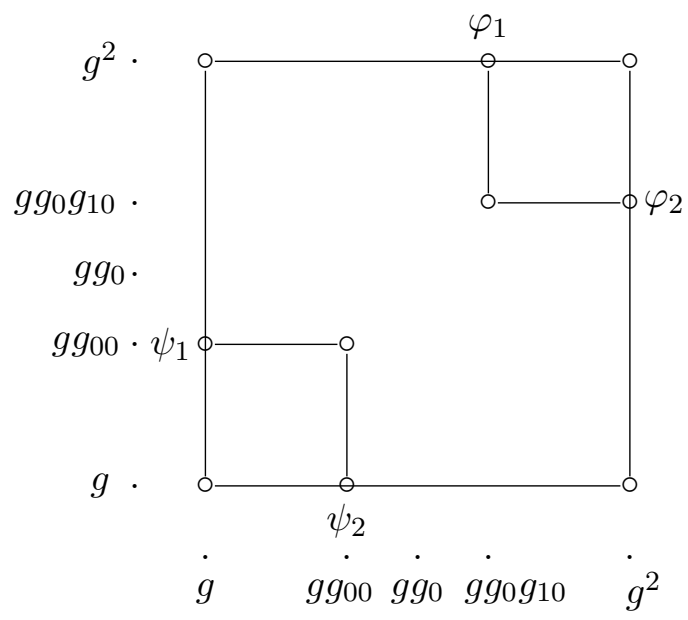

By trivial implications we see that all the formulas $\varphi_{1} \doteq \varphi_{g^{2}, g g_{0} g_{10}}, \varphi_{2} \doteq$ $\varphi_{g g_{0} g_{10}, g^{2}}, \psi_{1} \doteq \varphi_{g g_{00}, g}, \psi_{2} \doteq \varphi_{g, g g_{00}}$ belong to the square (that is $\varphi_{g^{2}, g} \rightarrow$ $\varphi_{i}, \psi_{j} \rightarrow \varphi_{g, g^{2}}$ for all $i$ and $j$ ). Let us check that the two diamonds on this diagram are incomparable. It suffices to check that $\psi_{1}$ does not imply $\varphi_{2}$ and that $\varphi_{1}$ does not imply $\psi_{2}$.

The first case is impossible, because by Lemma 3.3 the implication between formulae $\varphi_{a, b}$ cannot increase the divisibility condition given by $a$. Thus assume that $\varphi_{1}$ implies $\psi_{2}$, that is $\varphi_{g^{2}, g g_{0} g_{10}}$ implies $\varphi_{g, g g_{00}}$. Then by Lemma 3.4 we obtain $g B+g_{01} g_{10} B=B$. But $g_{01} g_{10}$ divides $g$, therefore $g B=B$, a contradiction.

Now clearly the above construction can be repeated for each small square, producing eventually an infinite sequence of squeezing incomparable diamonds in $L(B)$. Thus the lattice $L(B)$ has no width.

In the remaining part of this section we will prove the necessity in Theorem 7.1, that is the nonexistence of width of the lattice $L(B)$ implies that the value group $\Gamma(B)$ contains a dense subchain.

Suppose that a Bézout domain $B$ is such that the width of the lattice $L(B)$ is undefined. By the definition of width we can find a countable elementary subring $B^{\prime}$ of $B$ such that the width $L\left(B^{\prime}\right)$ is undefined. Because there is an obvious embedding from $\Gamma\left(B^{\prime}\right)$ to $\Gamma(B)$, it will be sufficient to prove that $\Gamma\left(B^{\prime}\right)$ contains a dense subchain. Furthermore, by Fact $6.1, B^{\prime}$ possesses a superdecomposable pure injective module, hence a superdecomposable type in one variable. In what follows we will analyze this type. Thus from the very beginning we may assume that $B$ has a superdecomposable pp-type.

Recall that a pp-type $p$ is said to be superdecomposable, if its pure injective envelope $N(p)$ is superdecomposable, that is contains no indecomposable 
direct summands. It follows from another result of Ziegler (see [14, Sect. 6]) that $p$ is superdecomposable if and only if $p$ contains no large formula. Since the lattice $L(B)$ for a Bézout domain $B$ is distributive, the formula $\varphi \in p^{-}$ is large if for all $\varphi_{1}, \varphi_{2} \in p^{-}$such that $\varphi \rightarrow \varphi_{1}, \varphi_{2}$ we have $\varphi_{1}+\varphi_{2} \in p^{-}$.

The following diagram illustrates this configuration:

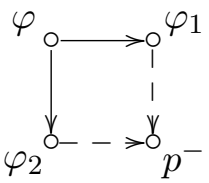

From Lemma 2.3 we easily derive

Lemma 7.2. Let $p$ be a nonzero pp-type over a Bézout domain B. Then $p$ is superdecomposable if and only if for any $\varphi_{a, b} \in p^{-}$there are $\varphi_{a_{1}, b_{1}}, \varphi_{a_{2}, b_{2}} \in$ $p^{-}$such that $\varphi_{a, b} \rightarrow \varphi_{a_{1}, b_{1}}, \varphi_{a_{2}, b_{2}}$ and $\varphi_{a_{1}, b_{1}}+\varphi_{a_{2}, b_{2}} \in p$.

Now we will translate this condition into the language of the function $F(p)$ corresponding to $p$ (see Proposition 5.2). As we will see, there exists a clear trichotomy for the behavior of this function.

Proposition 7.3. Let $p$ be a nonzero pp-type in one variable over a Bézout domain $B$ and let $F=F(p)$ be the corresponding function from $\Gamma^{+}$to $\widehat{\Gamma}^{+}$. Then $p$ is superdecomposable if and only if the following holds.

For every $a, b \in B$ such that $a \notin F(b)$, either

(i) there are $c, d \in B$ such that $c<a, b<d$, $a \notin F(d), c \notin F(b)$ and $c \in F(d):$
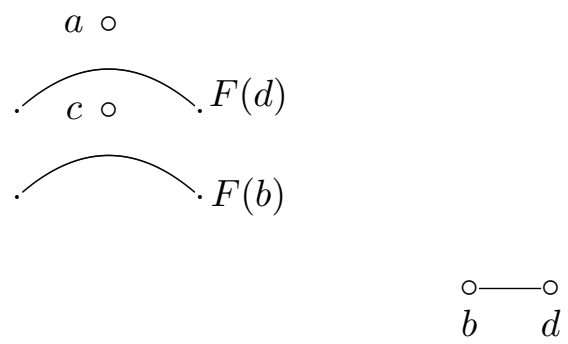

or

(ii) there are $e, g \in B$ such that $e, g<a, e, g \notin F(b)$ but $\operatorname{gcd}(e, g) \in F(b)$ :

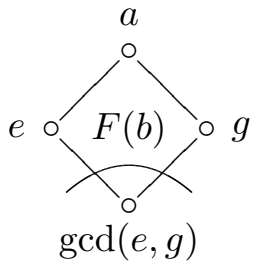


(iii) there are $f, h \in B$ such that $b<f, h, a \notin F(f), F(h)$ but $a \in$ $F(\operatorname{lcm}(f, h))$ :

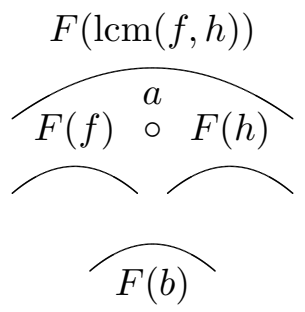

Note that the condition (i) has already occurred for valuation domains (see [9, Prop. 12.11]), however (ii) and (iii) cannot occur when $\Gamma(B)$ is linearly ordered.

Proof. $\Leftarrow$. By Lemma 7.2 it suffices to show that no formula $\varphi_{a, b} \in p^{-}$is large in $p$ (according to the statement of the lemma). Suppose first that (i) holds. Consider the following diagram:

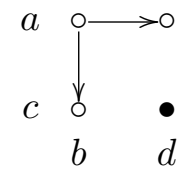

(here circles denote points in $p^{-}$and bullets formulas in $p$ ). Since $a \notin F(d)$ we obtain $\varphi_{a, d} \in p^{-}$, and similarly $c \notin F(b)$ implies $\varphi_{c, b} \in p^{-}$. Furthermore, $\varphi_{a, b} \rightarrow \varphi_{a, d}, \varphi_{c, b}$ are trivial implications, and $\varphi_{a, d}+\varphi_{c, b}=\varphi_{c, d} \in p$, because $c \in F(d)$.

Similarly in case (ii) we have trivial implications $\varphi_{a, b} \rightarrow \varphi_{e, b}, \varphi_{g, b}$, where the last two formulae are in $p^{-}$, because $e, g \notin F(b) . \operatorname{But} \operatorname{gcd}(e, g) \in F(b)$ yields $\varphi_{e, b}+\varphi_{g, b}=\varphi_{\operatorname{gcd}(e, g), b} \in p$.

The condition (iii) is analyzed similarly.

$\Rightarrow$. Let $a, b \in B$ be such that $a \notin F(b)$ but neither (i), (ii) or (iii) holds. We will show that the formula $\varphi_{a, b} \in p^{-}$is large in $p$ getting a contradiction. Thus we have to prove that for any $\varphi_{e, f}, \varphi_{g, h} \in p^{-}$such that $\varphi_{a, b}$ implies both these formulae, $\varphi_{e, f}+\varphi_{g, h} \in p^{-}$.

Suppose first that $a \neq 0$. By Lemma 3.4 the implication $\varphi_{a, b} \rightarrow \varphi_{e, f}$ yields $e \leq a$, and the elements $e$ and $\operatorname{lcm}(b, f) / f$ are coprime. We would like to replace $f$ by $f^{\prime}=\operatorname{lcm}(b, f)$, that is to force that $b \leq f$. Indeed $\varphi_{a, b}$ implies $\varphi_{e, f^{\prime}}$, because $f \leq f^{\prime}$. Furthermore, because $f^{\prime}=f \cdot\left(f^{\prime} / f\right)$ and $e$ and $f^{\prime} / f$ are coprime, the conditions 3$)$ and 4 ) of Proposition 5.2 imply that $e \in F(f)$ if and only if $e \in F\left(f^{\prime}\right)$, that is $\varphi_{e, f} \in p^{-}$if and only if $\varphi_{e, f^{\prime}} \in p^{-}$. 
Furthermore $\varphi_{e, f}+\varphi_{g, h} \rightarrow \varphi_{e, f^{\prime}}+\varphi_{g, h}$, so if the latter formula is in $p^{-}$, the same is true for the former formula.

Thus we can assume that $e \leq a, b \leq f$, and similarly that $g \leq a, b \leq h$. Now put $c=\operatorname{gcd}(e, g)$ and $d=\operatorname{lcm}(f, h)$, in particular $c \leq a$ and $b \leq d$.

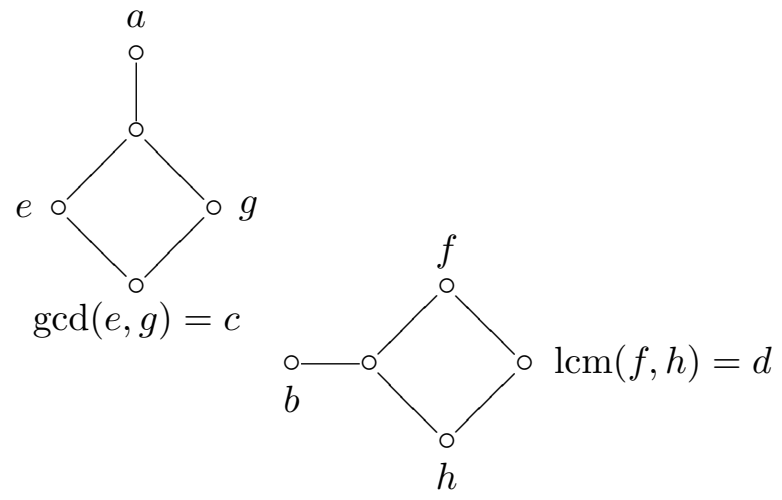

By $\neg($ i) we obtain that either

$\left({ }^{*} 1\right) c \in F(b)$, or

(*2) $a \in F(d)$, or

$\left({ }^{*} 3\right) c \notin F(d)$.

First assume $(* 1)$. We know that $e, g \leq a$ and $a \notin F(b)$. Furthermore $b \leq f$ and $e \notin F(f)$ imply $e \notin F(b)$; and $b \leq h$ and $g \notin F(h)$ yield $g \notin F(b)$. But then $c \in F(b)$ contradicts $\neg$ (ii).

Similarly if $\left(*^{*}\right)$ holds, then we have $b \leq f, h$ and $a \notin F(b)$. Furthermore $e \leq a$ and $e \notin F(f)$ imply $a \notin F(f)$; and $g \leq a$ and $g \notin F(h)$ yield $a \notin F(h)$. But then $a \in F(d)$ contradicts $\neg$ (iii).

Thus we are left with $\left({ }^{*} 3\right)$. Then $c \notin F(d)$ implies $\varphi_{c, d} \in p^{-}$. But $\varphi_{c, d}=\varphi_{e, f}+\varphi_{g, h}$, therefore this sum is in $p$, as desired.

The case $a=0$ is considered similarly.

Let us examine the various cases generated by conditions (i), (ii) and (iii) from Proposition 7.3.

Proposition 7.4. Let $B$ be a Bézout domain and let $p$ be a superdecomposable pp-type over $B$ such that every pair of elements $a, b \in B$ with $\varphi_{a, b} \in p^{-}$ satisfies (i). Then the lattice $\Gamma^{+}(B)$ contains a subchain isomorphic to the ordering $(\mathbb{Q}, \leq)$ of the rationals.

Proof. Take $a, b \in B$ with $\varphi_{a, b} \in p^{-}$. Look at the corresponding elements $c, d$ provided by (i). Then both $\varphi_{c, b}$ and $\varphi_{a, d}$ are not in $p$, therefore we can apply to $c, b$ and $a, d$ the same procedure. 
First let us deal with $c, b$. By (i) there are elements $c^{\prime}, d^{\prime} \in B$ such that $c^{\prime}<c, b<d^{\prime}$, and $\varphi_{c, d^{\prime}}, \varphi_{c^{\prime}, b} \in p^{-}$but $\varphi_{c^{\prime}, d^{\prime}} \in p$.

It follows easily that $c^{\prime}<a$ and we claim that we can also assume that $d^{\prime} \leq d$. To do that, just replace $d^{\prime}$ with $D \doteq \operatorname{gcd}\left(d, d^{\prime}\right)$. Then clearly $b \leq D \leq d:$

$$
\begin{aligned}
& a \circ \\
& c \circ \\
& c^{\prime} \circ
\end{aligned}
$$

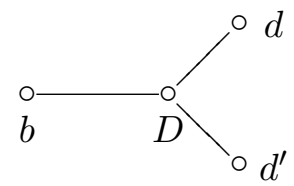

Furthermore $\varphi_{c, D} \in p^{-}$and $\varphi_{c^{\prime}, D} \in p$.

Namely note that $\varphi_{c, D} \in p$ combined with $D \leq d^{\prime}$ would imply $\varphi_{c, d^{\prime}} \in p$, a contradiction. Also observe that $c^{\prime} \leq c$ and $\varphi_{c, d} \in p$ imply $\varphi_{c^{\prime}, d} \in p$. Because $\varphi_{c^{\prime}, d^{\prime}} \in p$, Lemma 3.1 yields $\varphi_{c^{\prime}, D} \in p$.

Furthermore from $\varphi_{c, d} \in p$ and $\varphi_{c, D} \in p^{-}$we obtain $D<d$; and $\varphi_{c^{\prime}, D} \in p$ and $\varphi_{c^{\prime}, b} \in p^{-}$imply $b<D$.

In conclusion we may assume that $b<d^{\prime}<d$. The next diagram describes this part of the proof, where again by circles we denote points in $p^{-}$and by bullets formulas in $p$.

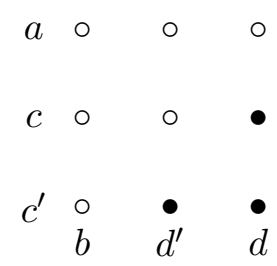

Similarly, when considering $a, d$, we will find elements $c^{\prime \prime}, d^{\prime \prime} \in B$ such that $c^{\prime \prime}<a, d<d^{\prime \prime}$, and $\varphi_{a, d^{\prime \prime}}, \varphi_{c^{\prime \prime}, d} \in p^{-}, \varphi_{c^{\prime \prime}, d^{\prime \prime}} \in p$.

$$
\begin{array}{ccc}
a & \circ & \circ \\
c^{\prime \prime} & \circ & \bullet \\
& d & d^{\prime \prime}
\end{array}
$$

Replacing $c^{\prime \prime}$ by $C \doteq \operatorname{lcm}\left(c^{\prime}, c^{\prime \prime}\right)$, we can also assume $c \leq c^{\prime \prime}$. Obviously $c \leq C \leq a$. 

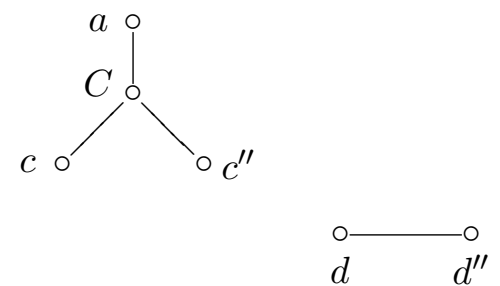

Furthermore $\varphi_{C, d} \in p^{-}$and $\varphi_{C, d^{\prime \prime}} \in p$.

Namely $\varphi_{C, d} \in p$ and $c^{\prime \prime} \leq C$ would imply $\varphi_{c^{\prime \prime}, d} \in p$, a contradiction. For the second condition note that $\varphi_{c, d} \in p$ and $d \leq d^{\prime \prime}$ gives $\varphi_{c, d^{\prime \prime}} \in p$. Together with $\varphi_{c^{\prime \prime}, d^{\prime \prime}} \in p$, by Lemma 3.1 this implies $\varphi_{C, d^{\prime \prime}} \in p$.

As a consequence, $\varphi_{c, d} \in p$ and $\varphi_{C, d} \in p^{-}$imply $c<C$; and $\varphi_{a, d} \in p$ and $\varphi_{C, d} \in p^{-}$yield $c<a$. Thus taking $C=c^{\prime \prime}$ we obtain:

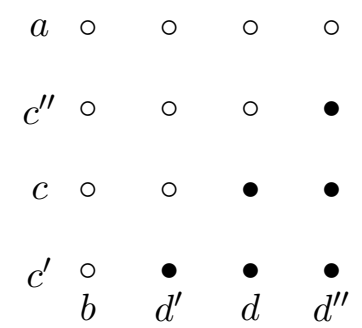

(where, as before, circles and bullets correspond to formulas in $p^{-}, p$ respectively). At this point we can repeat the same construction for formulas $\varphi_{c, d^{\prime}}, \varphi_{c^{\prime}, b}, \varphi_{a, d^{\prime \prime}}$ and $\varphi_{c^{\prime \prime}, d}$. As in $[9$, Thm. 12.11] this eventually generates two dense countable chains inside $\Gamma^{+}(B)$, the former containing $a, c^{\prime \prime}, c, c^{\prime}$, and the latter $b, d^{\prime}, d, d^{\prime \prime}$.

Thus we are led to consider Bézout domains $B$ and superdecomposable pp-types $p$ over $B$ such that some pair of elements $a, b \in B$ satisfies (ii) or (iii) but not (i).

The following remark has a straightforward proof.

Remark 7.5. 1) If $e \leq a$ and the pair (e,b) satisfied (i), then the same holds true for $(a, b)$.

2) If $b \leq f$ and $(a, f)$ satisfies (i), then the same is true for $(a, b)$.

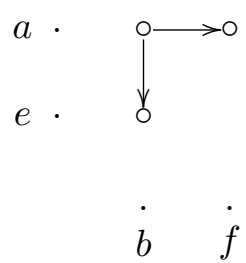


The following lemma shows that in the absence of (i), the conditions (ii) and (iii) are preserved when forming our basic configuration.

Lemma 7.6. Let $(a, b)$ be a pair of elements of $B$ for which (i) fails (and hence $(a, b)$ satisfies (ii) or (iii)).

1) Assume that $(a, b)$ satisfies (ii). Then, with notation of Proposition 7.3 $(e, b)($ or $(g, b))$ satisfies (iii) if and only if $(a, b)$ satisfies (iii).

2) Assume that $(a, b)$ satisfies (iii). Then, with notation of Proposition 7.3 $(a, f)$ (or $(a, h))$ satisfies (ii) if and only if $(a, b)$ satisfies (ii).

Proof. We will prove 2). The proof of 1) is similar.

Suppose first that $(a, f)$ satisfies (ii). Let $e^{\prime}, g^{\prime}$ witness that. Thus $e^{\prime}, g^{\prime} \leq$ $a, \varphi_{e^{\prime}, f}, \varphi_{g^{\prime}, f} \in p^{-}$and $\varphi_{C, f} \in p$, where $C$ denotes $\operatorname{gcd}\left(e^{\prime}, f^{\prime}\right)$.

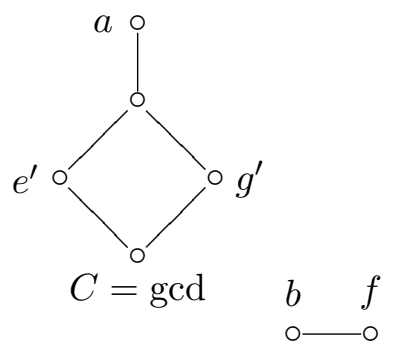

From $\varphi_{e^{\prime}, f}, \varphi_{g^{\prime}, f} \in p^{-}$and $b \leq f$ it follows that $\varphi_{e^{\prime}, b}, \varphi_{g^{\prime}, b} \in p^{-}$. If $\varphi_{C, b} \in p^{-}$, then the pair $(a, b)$ satisfies (i), a contradiction. Thus $\varphi_{C, b} \in p$ witnesses (ii) for $(a, b)$.

Conversely suppose that $(a, b)$ satisfies (ii). Then there are $e, g \leq a$ such that $\varphi_{e, b}, \varphi_{g, b} \in p^{-}$but $\varphi_{C, b} \in p$, where $C=\operatorname{gcd}(e, g)$. Then $\varphi_{C, b} \in p$ and $b \leq f$ implies $\varphi_{C, f} \in p$. If $\varphi_{e, f} \in p$, then the pair $(a, b)$ satisfies (i), a contradiction. Thus $\varphi_{e, f} \in p^{-}$, and similarly $\varphi_{g, f} \in p^{-}$. Thus we obtained a configuration (ii) for the pair $(a, f)$.

We will further analyze cases (ii) and (iii).

Theorem 7.7. Let $B$ a Bezout domain, $p$ be a superdecomposable pp-type over $B$ such that some pair of elements $(a, b)$ satisfies (ii) or (iii) but not (i) in Proposition 7.3. Then the lattice $\Gamma^{+}(B)$ includes either

A) a binary tree $a_{\eta}, \eta \in 2^{<\omega}$ such that

1) $a_{\emptyset}=a, \varphi_{a, b} \in p^{-}$,

2) if $\eta$ is a prefix of $\eta^{\prime}$, then $a_{\eta^{\prime}} \leq a_{\eta}$,

3) $a_{\eta 00}, a_{\eta 01} \geq \operatorname{gcd}\left(a_{\eta 0}, a_{\eta 1}\right)$ and $a_{\eta 10}, a_{\eta 11} \geq \operatorname{gcd}\left(a_{\eta 0}, a_{\eta 1}\right)$,

4) for all $\eta \in 2^{<\omega}$ we have $\varphi_{a_{\eta 0}, b}, \varphi_{a_{\eta 1}, b} \in p^{-}$but $\varphi_{\operatorname{gcd}\left(a_{\eta 0}, a_{\eta 1}\right), b} \in p$ 


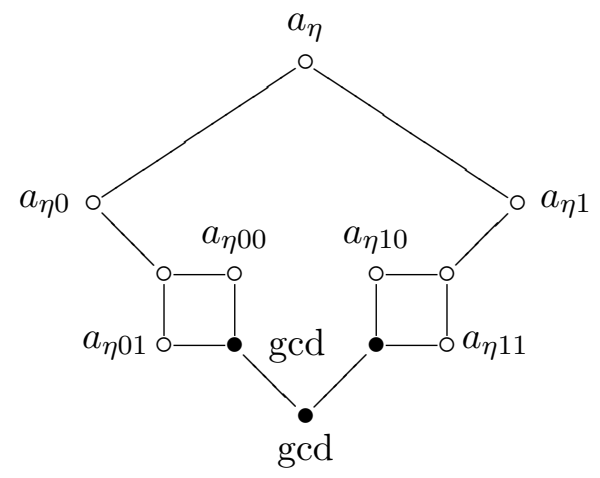

or

B) a binary tree $b_{\eta}, \eta \in 2^{<\omega}$ such that

1) $b_{\emptyset}=b, \varphi_{a, b} \in p^{-}$,

2) if $\eta$ is a prefix of $\eta^{\prime}$, then $b_{\eta} \leq b_{\eta^{\prime}}$,

3) $b_{\eta 00}, b_{\eta 01} \leq \operatorname{lcm}\left(b_{\eta 0}, b_{\eta 1}\right)$ and $b_{\eta 10}, b_{\eta 11} \leq \operatorname{lcm}\left(b_{\eta 0}, b_{\eta 1}\right)$,

4) for all $\eta \in 2^{<\omega}$ we have $\varphi_{a, b_{\eta 0}}, \varphi_{a, b_{\eta 1}} \in p^{-}$but $\varphi_{a, \operatorname{lcm}\left(b_{\eta 0}, b_{\eta 1}\right)} \in p$.

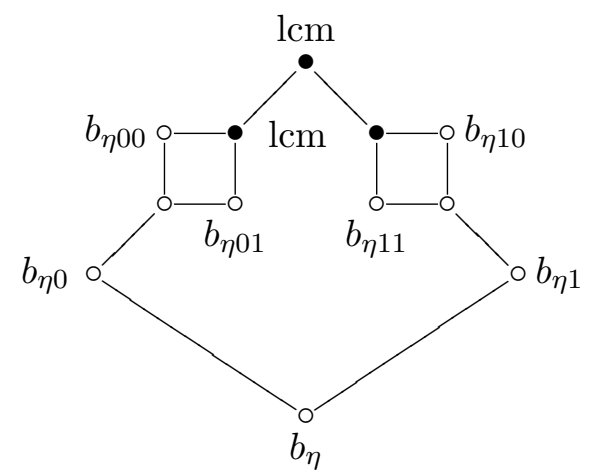

Proof. By symmetry we may assume that $(a, b)$ satisfies (ii) and we set $a_{\emptyset}=a$. Thus there are $a_{0}, a_{1}<a$ such that $\varphi_{a_{0}, b}, \varphi_{a_{1}, b} \in p^{-}$but for $c=\operatorname{gcd}\left(a_{0}, a_{1}\right)$ we have $\varphi_{c, b} \in p$, that is $\varphi_{a_{0}, b}+\varphi_{a_{1}, b} \in p$. Taking into account Lemma 7.6 by symmetry we may further assume that both $\left(a_{0}, b\right)$ and $\left(a_{1}, b\right)$ satisfy (ii). Thus choose $a_{00}^{\prime}, a_{01}^{\prime}<a_{0}$ such that $\varphi_{a_{00}^{\prime}, b}, \varphi_{a_{01}^{\prime}, b} \in p^{-}$ but for $c^{\prime}=\operatorname{gcd}\left(a_{00}^{\prime}, a_{01}^{\prime}\right)$ we have $\varphi_{c^{\prime}, b} \in p$. 


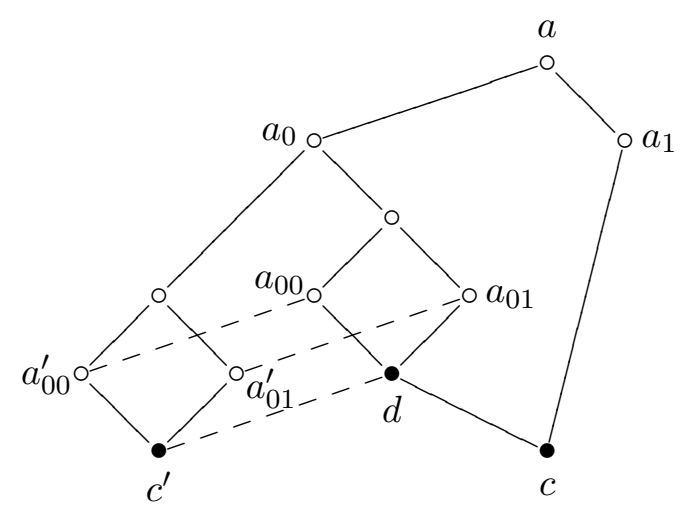

Now take $a_{00}=\operatorname{lcm}\left(a_{00}^{\prime}, c\right)$ and $a_{01}=\operatorname{lcm}\left(a_{01}^{\prime}, c\right)$. Using distributivity, it is not difficult to check that these elements are as desired. Namely note that $\varphi_{a_{00}^{\prime}, b} \in p^{-}$, therefore the conjunction of this formulas with $\varphi_{c, b}$ is in $p^{-}$. But the first coordinate of this conjunction is $\operatorname{lcm}\left(a_{00}^{\prime}, c\right)=a_{00}$. Thus $\varphi_{a_{00}, b} \in p^{-}$and similarly $\varphi_{a_{01}, b} \in p^{-}$. Now if we denote $d \doteq \operatorname{gcd}\left(a_{00}, a_{01}\right)$ then clearly $d=\operatorname{lcm}\left(c, c^{\prime}\right)$, hence $\varphi_{a_{00}, b}+\varphi_{a_{01}, b}=\varphi_{d, b}$ equals $\varphi_{c, b} \wedge \varphi_{c^{\prime}, b}$, therefore lies in $p$.

A similar construction applies to $a_{1}$ and so on. Thus we will eventually construct a binary tree as required in A).

Now we are in a position to complete the proof of Theorem 7.1. Indeed, the sufficiency has been already established. To prove the necessity arguing as above we may suppose that $B$ has a superdecomposable pp-type $p$. If every pair $(a, b)$ in $p^{-}$satisfies (i), then Proposition 7.4 produces a dense subchain in $\Gamma^{+}(B)$. Otherwise by Theorem 7.7 we obtain even more, namely that $\Gamma^{+}(B)$ contains even a 'squeezing diamonds' configuration (in particular has no width).

\section{Discussion}

First in this section we will give an example of a superdecomposable pptype over a Bézout domain $B$ such that each point $(a, b)$ not in $p$ satisfies (ii) but neither (i) nor (iii). Namely let $B=\mathbb{Z}^{\omega} / U$, where $U$ is any non-principal ultrafilter on $\omega$, in particular $B$ is elementarily equivalent to $\mathbb{Z}$. To any $U$ class of $B$, say of $a \in \mathbb{Z}^{\omega}$, we associate the number $c_{n}$ of different primes in the decomposition of the $n$-coordinate $a_{n}$ of $a$ into prime factors. Of course this definition is not sound but the property that $c_{n}$ goes to infinity as $n$ goes to infinity does not depend on the choice of the representative $a$, but only on the $U$-class. Such elements will be called large, and all the remaining elements of $B$ will be called small. 
Now include the formula $\varphi_{a, b}$ in $p^{-}$if $a$ is large, and take this formula in $p$ if $a$ is small, and proceed as usual to complete this set of formulas to a pp-type. It is not difficult to check that $p$ is consistent. Suppose that $\varphi_{a, b} \in p^{-}$. Clearly $a$ is a product of two large elements $c, d$ such that $c$ and $d$ are coprime. Then $\varphi_{a, b} \rightarrow \varphi_{c, b}, \varphi_{d, b} \in p^{-}$and $\varphi_{c, b}+\varphi_{d, b}=\varphi_{1, b} \in p$, as desired for (ii).

Because $p$ is completely determined by divisibility conditions, it is easily checked that no pair $(a, b) \in p^{-}$satisfies (i) or (iii). Note that the superdecomposable pure injective module $N(p)$ is additionally torsion-free.

Let us discuss briefly how difficult is to construct a superdecomposable pure injective module in case when a Bézout domain $B$ has a binary tree of elements $b_{\eta}$ satisfying condition (iii) from Theorem 7.7. In this case the theory of divisible $B$-modules has no width, so we could expect a superdecomposable pure injective model of this theory. Furthermore it is not difficult to check that such a module exists if and only if, for some ideal $I$ of $B$, the injective envelope of the cyclic module $B / I$ is superdecomposable. This gives the following condition on $I$ : for every $b \notin I$ there are $s, t \in B$ such that $b s, b t \notin I$, but $\operatorname{lcm}(b s, b t) \in I$. However we are not able to construct such an ideal just from the sequence $b_{\eta}$ with (iii), and the existence of such ideal seems to be a difficult problem.

Dually, we have a similar problem in case (ii), but now with superdecomposable pure injective torsion-free $B$-modules. The case (i) seems to be even harder to analyze.

However, because our analysis depends only on $\Gamma(B)$, this is a problem of the theory of lattice ordered abelian groups, which are classified (see [1, Sec. 3.4]). Thus we hope that the use of this theory will lead to its solution.

\section{REFERENCES}

[1] L. Fuchs, L. Salce, Modules over non-Noetherian Domains, Mathematical Surveys and Monographs Series, Vol. 84, American Mathematical Society, 2001.

[2] M. Golasiński, M. Henriksen, Residue class rings of real-analytic functions, Colloq. Math., 104 (2006), 85-97.

[3] L. Gregory, Ziegler spectra of valuation rings, PhD Thesis, University of Manchester, 2011.

[4] L. Gregory, Sobriety of the Ziegler spectrum of a Prüfer domain, J. Pure Appl. Algebra, to appear.

[5] L. Gregory, Decidability for theories of modules over valuation domains, Preprint.

[6] O. Helmer, Divisibility properties of integral functions, Duke Math. J., 6 (1940), 345-356. 
[7] M. Prest, Purity, Spectra and Localization, Encyclopedia of Mathematics and its Applications, Vol. 121, Cambridge University Press, 2009.

[8] G. Puninski, Cantor-Bendixson rank of the Ziegler spectrum over a commutative valuation domain, J. Symbolic Logic, 64 (1999), 1512-1518.

[9] G. Puninski, Serial Rings, Kluwer, 2001.

[10] G. Puninski, Krull-Gabriel dimension and Cantor-Bendixson rank of 1-domestic string algebras, Colloq. Math., 127 (2012), 185-210.

[11] G. Puninski, M. Prest, P. Rothmaler, Rings described by various purities, Comm. Algebra, 27 (1999), 2127-2162.

[12] G. Puninski, V. Puninskaya, C. Toffalori, Decidability of the theory of modules over commutative valuation domains, Ann. Pure Appl. Logic, 145 (2007), 258-275.

[13] R.B. Warfield, Purity and algebraic compactness for modules, Pacif. J. Math., 28 (1969), 699-719.

[14] M. Ziegler, Model theory of modules, Ann. Pure Appl. Logic, 26 (1984), 149-213.

(G. Puninski) Belarusian State University, Department of Mathematics, Praspekt Nezalezhnosti 4, 220030 Minsk, Belarus

E-mail address: punins@mail.ru

(C. Toffalori) University of Camerino, School of Science and Technologies, Division of Mathematics, Via Madonna delle Carceri 9, 62032 Camerino, Italy

E-mail address: carlo.toffalori@unicam.it 